\title{
NEXXUS: A comprehensive ROSAT survey of coronal X-ray emission among nearby solar-like stars ${ }^{\star}$
}

\author{
J. H. M. M. Schmitt and C. Liefke
}

\author{
Hamburger Sternwarte, Universität Hamburg, Gojenbergsweg 112, 21029 Hamburg, Germany \\ e-mail: cliefke@hs .uni-hamburg.de
}

Received 19 February 2003 / Accepted 20 March 2003

\begin{abstract}
We present a final summary of all ROSAT X-ray observations of nearby stars. All available ROSAT observations with the ROSAT PSPC, HRI and WFC have been matched with the CNS4 catalog of nearby stars and the results gathered in the Nearby $\mathbf{X}$-ray and XUV-emitting Stars data base, available via www from the Home Page of the Hamburger Sternwarte at the URL http://www.hs. uni-hamburg.de/DE/For/Gal/Xgroup/nexxus. New volume-limited samples of F/G-stars $\left(d_{\lim }=14 \mathrm{pc}\right), \mathrm{K}$-stars $\left(d_{\lim }=12 \mathrm{pc}\right)$, and M-stars $\left(d_{\lim }=6 \mathrm{pc}\right)$ are constructed within which detection rates of more than $90 \%$ are obtained; only one star (GJ 1002) remains undetected in a pointed follow-up observation. F/G-stars, K-stars and M-stars have indistinguishable surface X-ray flux distributions, and the lower envelope of the observed distribution at $F_{\mathrm{X}} \approx 10^{4} \mathrm{erg} / \mathrm{cm}^{2} / \mathrm{s}$ is the X-ray flux level observed in solar coronal holes. Large amplitude variations in X-ray flux are uncommon for solar-like stars, but maybe more common for stars near the bottom of the main sequence; a large amplitude flare is reported for the M star LHS 288. Long term X-ray light curves are presented for $\alpha$ Cen A/B and Gl 86, showing variations on time scales of weeks and demonstrating that $\alpha \mathrm{Cen} \mathrm{B}$ is a flare star.
\end{abstract}

Key words. stars: activity - stars: coronae - stars: late-type - X-rays: stars

\section{Introduction}

The discovery of X-ray emission from normal stars with the Einstein Observatory (Vaiana et al. 1981) and ROSAT demonstrated the ubiquity of hot coronae around late-type stars. Specifically, the volume-limited surveys by Schmitt et al. (1995) and Schmitt (1997) showed that coronal formation is universal in the sense that coronae are formed basically for all (or almost all) main-sequence stars with outer convection zones. Furthermore, the state of "minimum energy" of a stellar corona (around a main sequence star) was found to be one whose mean X-ray surface flux corresponds to the surface flux of a solar coronal hole. Hünsch et al. (1999) and Sterzik \& Schmitt (1997) extended those studies to all stars within $25 \mathrm{pc}$ by cross-correlating the ROSAT all-sky survey (RASS) data with entries in the Gliese catalog (CNS3) of nearby stars and detected a significant fraction of stars listed in CNS3 as X-ray sources in the all-sky survey data, while Hünsch et al. (1998b) and Hünsch et al. (1998a) carried out similar studies for bright main sequence and giant stars in the Bright Star Catalog and Schröder et al. (1998) presented a volume-limited sample of

Send offprint requests to: J. H. M. M. Schmitt,

e-mail: jschmitt@hs.uni-hamburg.de

* Tables 1-3 are also available in electronic form at the CDS via anonymous ftp to cdsarc.u-strasbg.fr $(130.79 .128 .5)$ or via http://cdsweb.u-strasbg.fr/cgi-bin/qcat?]/A+A/417/651 giant stars within $35 \mathrm{pc}$ around the Sun. Similar studies on early-type stars were presented by Berghöfer et al. (1997), who present a catalog of bright O- and B-type stars detected in the ROSAT all-sky survey, while Fleming et al. (1996) discuss Xray emitting white dwarfs detected in the ROSAT all-sky survey. The dearth of X-ray emission among A-type stars has already been noted on the basis of observations with the Einstein Observatory (Schmitt et al. 1985), Hünsch (2001) presents a similar study from the ROSAT all-sky survey data, and Simon et al. (1995) from pointed ROSAT data. As to open clusters, most of them are too distant for sensitive studies with ROSAT survey data, so most of the ROSAT work on open clusters was carried using pointing data; for an overview of this work see Randich (2000). The Hyades cluster, however, has very a large angular extent because of its proximity and can be comprehensively studied only with survey data. Stern et al. (1995) present the as of to date most complete X-ray study of the Hyades, while Schmitt et al. (1993) present the ROSAT all-sky survey data on the core of the Pleiades cluster.

One of the key properties of late-type stars is their $\mathrm{X}$-ray variability. The ROSAT all-sky survey with its approximately $30 \mathrm{~s}$ snapshot exposures extending over two days and longer provided a unique sampling pattern of coronal $\mathrm{X}$-ray emission. In particular, long-duration flares and rotational modulation can be well studied with such data. Schmitt (1994) discusses ROSAT survey observations of flare stars, and 
Haisch \& Schmitt (1994) study specifically the X-ray variability of giants observed during the all-sky survey. Fuhrmeister \& Schmitt (2003) present a systematic study of X-ray variability in the RASS data, finding that stars are indeed the most variable class of X-ray emitters.

Meanwhile the ROSAT operations have ended and no further ROSAT data will be taken. Data from numerous individual pointings in particular with the ROSAT HRI have become available in the ROSAT archive, the ROSAT survey data have been reprocessed, and the Gliese catalog of nearby stars has been substantially updated. The purpose of this paper is therefore to revisit and supplement the studies by Schmitt (1997) and Sterzik \& Schmitt (1997) by utilizing all available - and presumably final since reprocessed - ROSAT data. Specifically, Schmitt et al. (1995), Schmitt (1997) and Sterzik \& Schmitt (1997) had to use the pre-HIPPARCOS distance scale, but far more accurate HIPPARCOS parallaxes are now available for many nearby stars. Interestingly, the parallaxes for the brighter stars tended to be "older" hence less reliable so that the composition of the volume-limited samples has changed. Further, the ongoing infrared all-sky surveys provide "new" stars even in the immediate solar vicinity. Especially for K-type stars more ROSAT data on nearby stars were taken during the last years of its lifetime. In summary, now appears to be a good opportunity for a definitive summary of all ROSAT observations of nearby stars.

\section{Observations}

The ROSAT Observatory was operated between 1990-1998. Between July 1990 and January 1991 it carried out its ROSAT All-Sky Survey (RASS) with the ROSAT Position Sensitive Proportional Counter (PSPC). Afterwards pointed observations of individual X-ray sources were carried out in the framework of the ROSAT guest investigator program both with the PSPC and a High (angular) Resolution Imager (HRI). These detectors had fields of view of about $7000 \mathrm{arcmin}^{2}$ for the PSPC and $1000 \mathrm{arcmin}^{2}$ for the HRI, so that many X-ray sources were picked up serendipitously in the field of view of many observations whose original scientific goal was actually quite different. A boron filter could be placed in front of the PSPC detector for pointed observations allowing to separate the X-ray band below $0.28 \mathrm{keV}$ (i.e., the "carbon" band) into two separate energy bands; however, only a rather small number of observations was carried out with the boron filter in place.

\subsection{X-ray data}

The results of both the RASS observations and the ROSAT pointed observations are available in the ROSAT results archive in the form of source lists. All five source catalogs used for this study, i.e. the ROSAT Bright Source and Faint Source Catalog, the Second ROSAT Source Catalog of Pointed Observations with the PSPC with and without filter and the First ROSAT Source Catalog of Pointed Observations with the HRI as well as detailed information on the detection and screening procedures applied in the construction of the catalogs are available via www from the ROSAT Home
Page at Max-Planck-Institut für Extraterrestrische Physik http://wave. xray.mpe.mpg.de/rosat/catalogue or its mirror sites. These catalogs were our primary source of information, and only in individual cases (discussed explicitly in our paper) did we go back to the original X-ray data. It is important to realize in this context that the above source catalogs have been constructed with rather conservative detection thresholds. The use of these conservative thresholds was mandatory since $\mathrm{X}$-ray sources were searched for everywhere in all the ROSAT images. Since we are interested in X-ray emission only from specific locations (i.e., at the positions of nearby stars), we are working with a far smaller number of trial positions and can therefore "afford" choosing lower acceptance thresholds without compromising on the number of spurious sources. It is therefore possible in principle to obtain X-ray "detections" from stars not listed in the above catalogs and in our data base; specific cases discussed below are the nearby K star Gl 653 and the nearby M-dwarf LHS 288.

\subsection{Optical data}

As our source of optical data we used the CNS4 compilation of nearby stars compiled by Jahreiss (2002). As pointed out above, the ongoing ever more sensitive infrared surveys provide "new" stars in the immediate vicinity of the Sun such as the M9V dwarf DENIS-P J104814.7-395606.1 at a distance of possibly 4.1 $\pm 0.6 \mathrm{pc}$ (Delfosse et al. 2001) or LHS 2090 (Scholz et al. 2001), although very often the parallax information on those stars is extremely limited. Therefore no compilation of nearby stars can be truly complete. Still, among the F, G and $\mathrm{K}$ type stars the CNS4 catalog ought to be complete and the only source of error should be the parallax error which can move a given star outside or inside a specified sampling volume. On the other hand, among the very late $\mathrm{M}$ stars and brown dwarfs the CNS4 catalog is bound to be incomplete.

\section{Data analysis}

The ROSAT source lists and the CNS4 catalog were searched for positional coincidences. As a matching criterion we used 120 arcsec for survey data, 60 arcsec for pointing data with the PSPC and 30 arcsec for HRI data. The differently chosen positional acceptance thresholds reflect the fact that, first, the intrinsic positional accuracy of survey data, and the PSPC and HRI pointing data decreases in that order, and second, the CNS4 input catalog does not have the most accurate positional information since it is not intended to be a positional catalog. The chosen acceptance thresholds do not introduce significant errors into our X-ray source lists. In quite a few cases multiple detections of coronal X-ray emission from individual stars are available. Specifically, many of the nearby stars observed and detected made in the ROSAT pointing program were also detected in the survey data.

We stress that X-ray source identifications are made solely on the basis of positional coincidence. Nevertheless we expect the number of spurious identifications to be very small. Calculating the number of identifications obtained by distributing approximately 100000 RASS sources over 3231 positions 
(i.e., the number of Gliese stars) with a detect cell radius of 2 arcmin, results in $\sim 27$ spurious identifications or $2 \%$ of the total number of RASS detections of nearby stars. The actual distribution of position offsets is much narrower. To be specific, only 16 out of 1217 survey detections have position offsets of more than 100 arcsec, and only $80 \mathrm{X}$-ray detections are off by more than 60 arcsec from the optical positions. We thus conclude that the fraction of incorrectly identified X-ray emitters is at the one percent level at worst.

\section{The NEXXUS data base}

Altogether we can associate 1333 of the 3231 stars up to a distance of 25 pc contained in the CNS4 catalog with ROSAT detected X-ray sources; the vast majority of these sources comes from the RASS data (1217). The remaining 116 stars were only detected in pointed observations. In addition to the survey detections, 328 stars were also observed with the PSPC in pointed mode without the boron filter, 49 with the filter, and 242 stars were observed with the ROSAT HRI. Moreover, some of these stars were observed more than once, so that multiple detections of coronal X-ray emission are available. For easy access and future reference the results of this cross-correlation process were assembled in the Nearby $\mathbf{X}$-ray and XUV-emitting Stars data base, available via www from the Home Page of the Hamburger Sternwarte at the URL http://www.hs. uni-hamburg.de/DE/For/Gal/Xgroup/ nexxus. For each CNS4 star detected in X-rays the database provides detailed information about the star itself, e.g. several catalog names, coordinates, proper motion, apparent and absolute magnitude, color indices, parallax and distance; and information about the associated X-ray source(s), e.g. coordinates, count rates with errors, background, exposure time, and an X-ray luminosity. For PSPC observations hardness ratios and a source likelihood are given, for HRI observations a signal to noise ratio. The date of observation, an off-axis angle, and source and sequence numbers of pointed observations are listed, too. To facilitate browsing, NEXXUS can be searched by coordinates, catalog name, color index, magnitude, proper motion or distance. For completeness we also list the XUV measurements of the CNS4 stars obtained with the ROSAT Wide Field Camera (WFC); the WFC data were kindly made available to NEXXUS by J. Pye (Leicester University). The WFC survey data is published by Pye et al. (1995), the pointed WFCdata will be discussed by Pye et al. (2003, in preparation).

\section{Results}

From the above cited numbers the overall ROSAT detection rate of the nearby stars listed in CNS4 is $41.1 \%$. It is instructive to compare a color-magnitude diagram of all CNS4 stars (cf., Fig. 1) with a similar diagram constructed only for NEXXUS stars (cf., Fig. 2). One immediately notes the wellknown paucity of X-ray emitting white dwarfs as well as the absence of X-ray detected brighter giants. Stellar X-ray emission is detected down to an absolute magnitude of $M_{V}=20$, i.e., down to the very bottom of the main sequence.

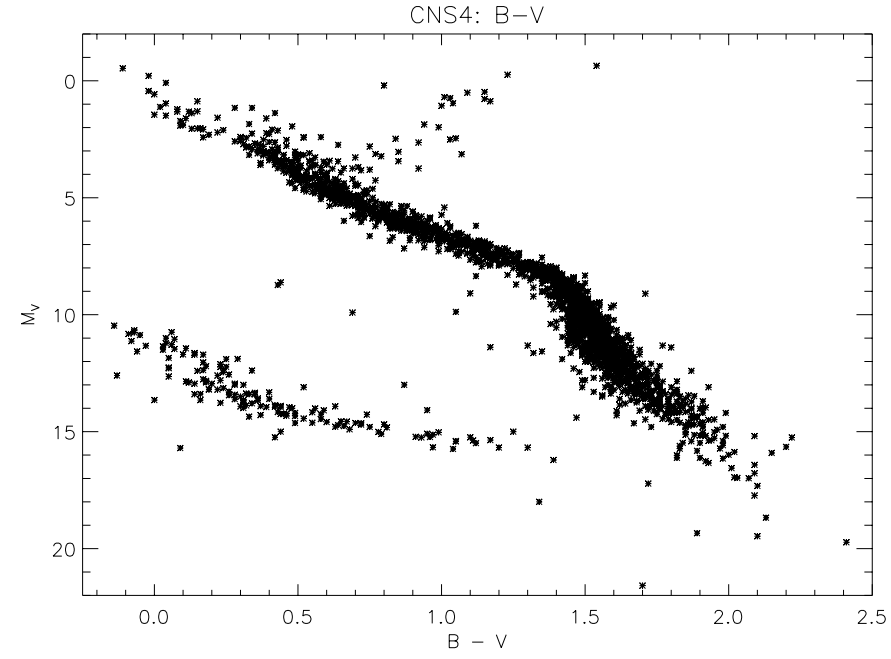

Fig. 1. Color-magnitude diagram $\left(B-V\right.$ vs. $\left.M_{V}\right)$ for all stars contained in CNS4 catalog.

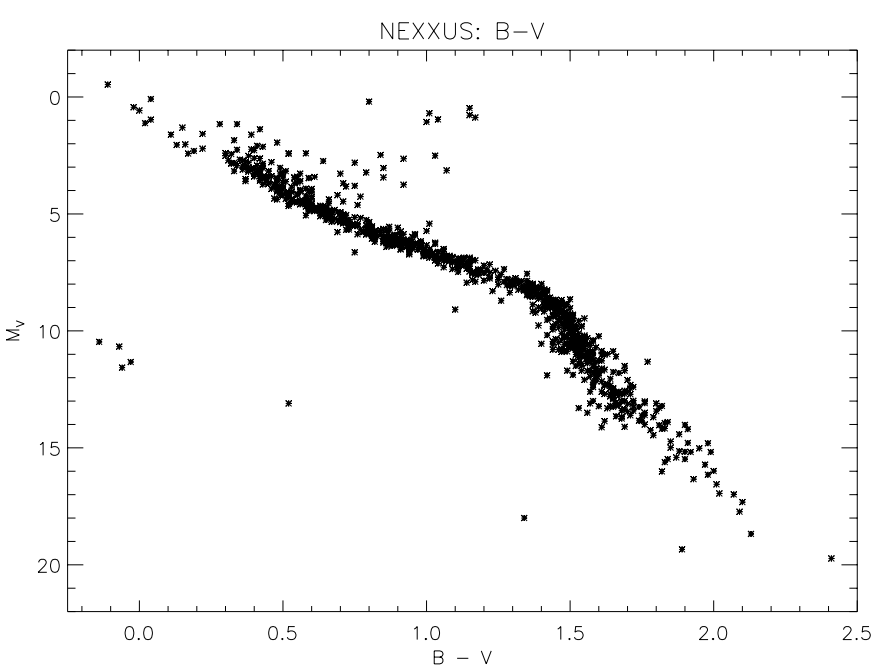

Fig. 2. Color-magnitude diagram ( $B-V$ vs. $M_{V}$ ) for all X-ray detected stars listed in the NEXXUS data base.

Except for white dwarfs and the brighter giants the overall detection rate of $41.1 \%$ is due to the lack of sufficient sensitivity for the more distant CNS4 stars. In the following we therefore focus on the very nearest stars for which almost complete detections are available. For this purpose we introduced three categories of stars. Since color information and spectral type are not always consistent, we decided to use a criterion based on absolute magnitudes to group our sample stars into three categories, those with $M_{V}$ in the range $3 \leq M_{V} \leq 5.80$ i.e., the F/G-stars, those with $5.80<M_{V} \leq 8.50$, i.e., the $\mathrm{K}$ stars, and those fainter than $M_{V}=8.50$, i.e., the M-type stars. We explicitly excluded all giants or white dwarfs from these samples; specifically, the giant stars Capella (Gl 194AB), a well-known RS CVn-like binary, $\beta$ Gem (=Gl 286; Hünsch et al. 1998a) and Arcturus (=Gl 541; Ayres et al. 1991) are not listed here. Table 1 contains the results for the F/G-stars, Table 2 those for the K stars, and Table 3 those for stars fainter than $M_{V}=8.50$. In each table we provide the stars' names, their absolute magnitude, their spectral type and distance as 


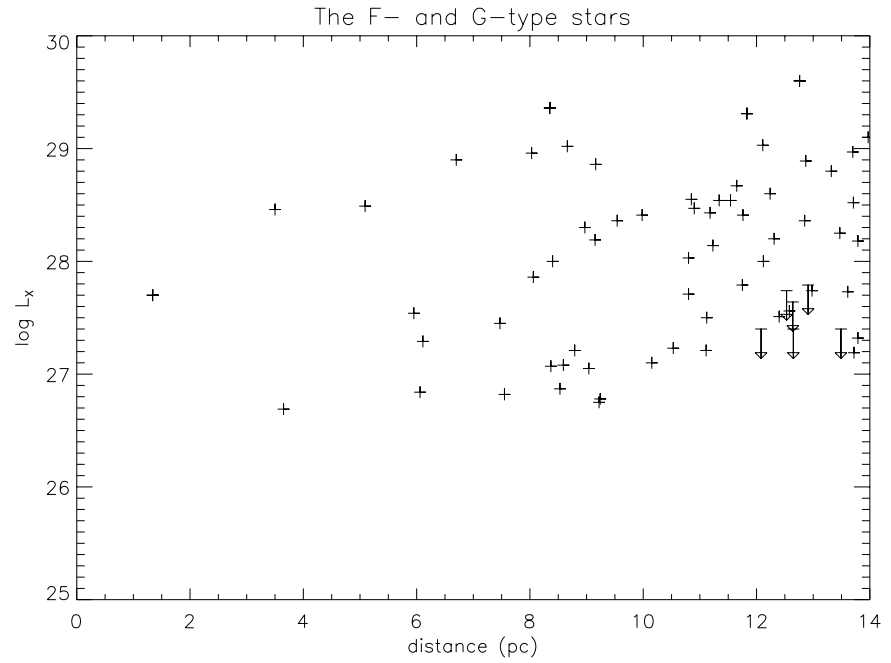

Fig. 3. $L_{\mathrm{X}}$ vs. distance for the $\mathrm{F} / \mathrm{G}$ star sample; note the complete sampling out to $12 \mathrm{pc}$.

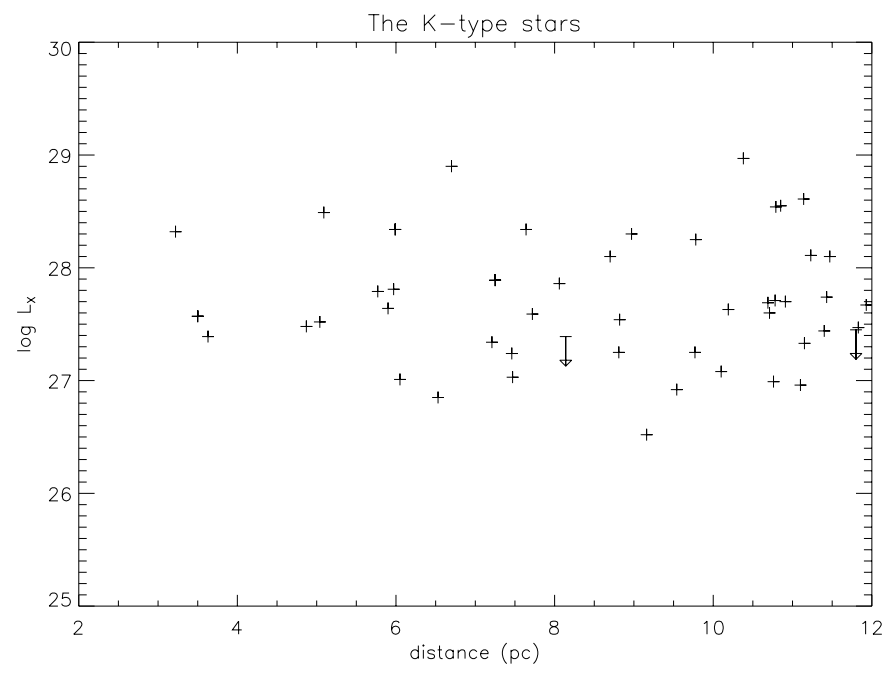

Fig. 4. $L_{\mathrm{X}}$ vs. distance for the $\mathrm{K}$ star sample; note the complete sampling out to $8 \mathrm{pc}$; the two upper limits come from survey data.

listed in the CNS4 catalog provided by Jahreiss (2002). For each star we then list the measured count rate and its error, the exposure time and an X-ray luminosity. In order to convert from count rate to $\mathrm{X}$-ray flux we used a conversion factor of $6 \times 10^{-12} \mathrm{erg} \mathrm{cm}^{-2} \mathrm{ct}^{-1}$ for PSPC data, $3 \times 10^{-11} \mathrm{erg} \mathrm{cm}^{-2} \mathrm{ct}^{-1}$ for PSPC with boron filter and $2.4 \times 10^{-11} \mathrm{erg} \mathrm{cm}^{-2} \mathrm{ct}^{-1}$ for HRI data; X-ray luminosities were compiled from these X-ray fluxes and the HIPPARCOS distances. For RASS and PSPC pointing data the source likelihood is given, for HRI data the signal to noise ratio of the detection. A flag indicates the source of the data; S stands for RASS data, P for PSPC pointing data without the boron filter, F for PSPC pointing data with the boron filter, and $\mathrm{H}$ for HRI data. If a star was observed more than once with the same instrumental setup, only the observation with the longest exposure time is listed. In the case of binaries the components are listed separately if they were resolved in any of the ROSAT observations, only one entry is given for binaries unresolved by ROSAT. As far as the X-ray data are

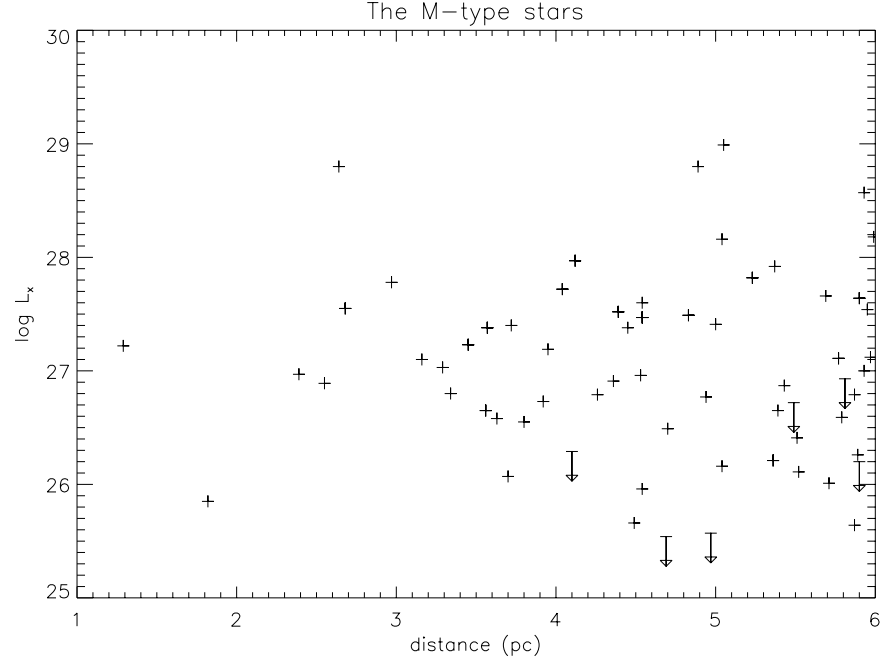

Fig. 5. $L_{\mathrm{X}}$ vs. distance for the $\mathrm{M}$ star sample.

concerned, Tables 1-3 are meant to replace Table 1 in Schmitt et al. (1995) and Table 2 in Schmitt (1997).

\subsection{Detection completeness}

In Figs. 3-5 we plot for the F/G-types stars, the K-type stars and the M-type stars the X-ray luminosity (in erg/s) as a function of distance (in pc). Inspection of Fig. 3 shows that among $69 \mathrm{~F} / \mathrm{G}$ stars within a distance of $14 \mathrm{pc}$ around the Sun, only seven (i.e., the stars Gl 67A, Gl 138, Gl 197, Gl 324A, Gl 354A, Gl 454 and Gl 541A) remained undetected. Note that in order to avoid multiple marks for a star observed more than once, only the observation with the longest exposure time was used to create the figures. The detection rate within the volume out to $14 \mathrm{pc}$ is therefore $94 \%$, and all stars within $12 \mathrm{pc}$ have been detected.

The $\mathrm{K}$ stars are plotted in Fig. 4; only two stars (i.e, Gl 776.3 and Gl 884) out of 51 have not been detected and hence the detection rate is $96 \%$. We remark in this context that the automatic analysis failed to detect the star Gl 653 in an HRI pointing. We therefore retrieved the original $\mathrm{X}$-ray data and generated an X-ray image having screened the photons to the pulse height range between channels 2-10. Inspection of this image shows a rather weak source within less than two arcsec of the expected proper motioned star position. Within a square box of 10 arcsec by $10 \operatorname{arcsec} 12$ counts were recorded with 6.1 being attributed to background; the remaining counts were then attributed to Gl 653. Obviously, a confirmation of this detection is highly desirable.

The M stars are plotted in Fig. 5; out to a distance of 6 pc 6 stars out of 65 have not been detected; the non-detected stars are Gl 570D, Gl 693, GJ 1002, LP 816-60, LP 944-20 and DENIS 1048-39. G1 570D is a brown dwarf not detected in an HRI pointing of the Gl 570 system; LP 944-20 is another brown dwarf undetected in a series of HRI pointings (Neuhäuser et al. 1999), but with a flare detected in a Chandra observation (Rutledge et al. 2000). Excluding those brown dwarfs all the derived upper limits except for the star GJ 1002 
Table 1. Sample stars with absolute magnitude between 3.00 and 5.80 (and some F-type stars brighter than 3.00 ) and a distance up to 14 pc, the F- and G-type stars. Table contains the star's name (Col. 1), its absolute visual magnitude (Col. 2), and its spectral type (Col. 3) as in CNS4; the distance (in pc, Col. 4) is computed from the CNS4 parallax. Mode (Col. 5) denotes the source of the X-ray data, S denoting survey data, P pointed PSPC data, and H pointed HRI data. The countrate (Col. 6) and its error (Col. 7) are quoted in cts/s, the likelihood or SNR are dimensionless (Col. 8), the exposure time (Col. 9) is in seconds, and the X-ray luminosity (Col. 10) in cgs-units.

\begin{tabular}{|c|c|c|c|c|c|c|c|c|c|}
\hline $\begin{array}{l}\text { Catalogue } \\
\text { name }\end{array}$ & $M_{V}$ & $\begin{array}{l}\text { Spectral } \\
\text { type }\end{array}$ & $\begin{array}{r}\text { Distance } \\
(\mathrm{pc})\end{array}$ & Mode & Count rate & Error & $\begin{array}{r}\text { Likelihood } \\
\text { or } \mathrm{S} / \mathrm{N}\end{array}$ & $\begin{array}{r}\text { Exposure } \\
\text { time }\end{array}$ & $\log L_{X}$ \\
\hline \multirow[t]{3}{*}{ Gl 5} & 5.44 & K0 V e & 13.70 & $\mathrm{~S}$ & 0.686800 & 0.048430 & 624.0 & 327 & 28.97 \\
\hline & & & & $\mathrm{P}$ & 0.793700 & 0.017800 & 3690.0 & 2575 & 29.03 \\
\hline & & & & $\mathrm{F}$ & 0.190600 & 0.005840 & 8385.0 & 5806 & 29.11 \\
\hline Gl 17 & 4.55 & F9 V & 8.59 & $\mathrm{P}$ & 0.022820 & 0.003500 & 75.0 & 2385 & 27.08 \\
\hline \multirow[t]{4}{*}{ Gl 19} & 3.42 & G2 IV & 7.47 & $\mathrm{~S}$ & 0.069990 & 0.023200 & 15.0 & 195 & 27.45 \\
\hline & & & & $\mathrm{P}$ & 0.038040 & 0.004100 & 114.0 & 2668 & 27.18 \\
\hline & & & & $\mathrm{F}$ & 0.010850 & 0.002680 & 26.0 & 1667 & 27.34 \\
\hline & & & & $\mathrm{H}$ & 0.037140 & 0.001630 & 21.0 & 14526 & 27.77 \\
\hline Gl 27 & 5.64 & K0 V & 11.11 & $\mathrm{H}$ & 0.004537 & 0.000952 & 4.9 & 6405 & 27.21 \\
\hline \multirow[t]{2}{*}{ Gl 34A } & 4.57 & G3 V & 5.95 & $\mathrm{~S}$ & 0.137900 & 0.021990 & 71.0 & 381 & 27.54 \\
\hline & & & & $\mathrm{H}$ & 0.026470 & 0.002070 & 10.2 & 6391 & 27.43 \\
\hline Gl 53A & 5.78 & G5 VI & 7.55 & $\mathrm{H}$ & 0.004004 & 0.000662 & 6.0 & 12750 & 26.82 \\
\hline Gl 61 & 3.44 & F8 V & 13.47 & $\mathrm{~S}$ & 0.134900 & 0.022790 & 66.0 & 354 & 28.25 \\
\hline Gl 67A & 4.44 & G1.5 V & 12.64 & $\mathrm{~S}$ & $<0.03801$ & & 2.1 & 377 & 27.64 \\
\hline \multirow[t]{2}{*}{ Gl 71} & 5.68 & G8 Vp & 3.65 & $\mathrm{~S}$ & 0.051750 & 0.013440 & 24.0 & 445 & 26.69 \\
\hline & & & & $\mathrm{H}$ & 0.009015 & 0.002360 & 3.9 & 2000 & 26.54 \\
\hline Gl 75 & 5.64 & $\mathrm{~K} 0 \mathrm{~V}$ & 9.98 & $\mathrm{~S}$ & 0.360200 & 0.026490 & 505.0 & 572 & 28.41 \\
\hline Gl 92A & 4.85 & G0 V e & 10.85 & S & 0.423400 & 0.039160 & 285.0 & 299 & 28.55 \\
\hline \multirow[t]{2}{*}{ Gl 107A } & 3.87 & F7 V & 11.23 & $\mathrm{~S}$ & 0.152400 & 0.018690 & 140.0 & 527 & 28.14 \\
\hline & & & & $\mathrm{H}$ & 0.016830 & 0.002590 & 5.5 & 2850 & 27.79 \\
\hline \multirow[t]{3}{*}{ Gl 111} & 3.72 & F6 V & 13.97 & $\mathrm{~S}$ & 0.906300 & 0.059260 & 559.0 & 288 & 29.10 \\
\hline & & & & $\mathrm{P}$ & 0.721600 & 0.020700 & 3473.0 & 1779 & 29.00 \\
\hline & & & & $\mathrm{F}$ & 0.167500 & 0.008840 & 4878.0 & 2136 & 29.07 \\
\hline \multirow[t]{3}{*}{ Gl 124} & 3.93 & G0 V & 10.53 & $S$ & 0.021440 & 0.007394 & 13.0 & 523 & 27.23 \\
\hline & & & & $\mathrm{P}$ & 0.058940 & 0.006000 & 145.0 & 1821 & 27.67 \\
\hline & & & & $\mathrm{H}$ & 0.010690 & 0.001140 & 8.8 & 9309 & 27.53 \\
\hline Gl 136 & 5.10 & $\mathrm{G} 2 \mathrm{~V}$ & 12.12 & $\mathrm{~S}$ & 0.095070 & 0.020590 & 35.0 & 328 & 28.00 \\
\hline \multirow[t]{4}{*}{ Gl 137} & 5.02 & $\mathrm{G} 5 \mathrm{~V}$ e & 9.16 & S & 1.201000 & 0.094630 & 472.0 & 286 & 28.86 \\
\hline & & & & $\mathrm{P}$ & 0.917700 & 0.024400 & 3994.0 & 1594 & 28.74 \\
\hline & & & & $\mathrm{F}$ & 0.285900 & 0.011600 & 4366.0 & 2144 & 28.94 \\
\hline & & & & $\mathrm{H}$ & 0.336300 & 0.005450 & 52.8 & 11246 & 28.91 \\
\hline Gl 138 & 4.81 & G1 V & 12.08 & $\mathrm{~S}$ & $<0.02417$ & & 0.5 & 338 & 27.40 \\
\hline \multirow[t]{3}{*}{ G1 139} & 5.35 & G5 V & 6.06 & $S$ & 0.025950 & 0.009569 & 10.0 & 519 & 26.84 \\
\hline & & & & $\mathrm{P}$ & 0.020170 & 0.004010 & 39.0 & 1738 & 26.73 \\
\hline & & & & $\mathrm{H}$ & 0.003099 & 0.000932 & 3.6 & 5913 & 26.51 \\
\hline Gl 147 & 3.59 & F8 V & 13.72 & $\mathrm{P}$ & 0.011510 & 0.002340 & 15.0 & 2809 & 27.19 \\
\hline \multirow[t]{2}{*}{ G1 150} & 3.75 & K0 IVe & 9.04 & $S$ & 0.019220 & 0.008239 & 9.0 & 532 & 27.05 \\
\hline & & & & $\mathrm{P}$ & 0.030400 & 0.002490 & 178.0 & 6383 & 27.25 \\
\hline Gl 177 & 4.86 & G1 V & 13.32 & S & 0.490200 & 0.045960 & 272.0 & 539 & 28.80 \\
\hline G1 178 & 3.66 & F6 V & 8.03 & $\mathrm{~S}$ & 1.957000 & 0.146700 & 481.0 & 392 & 28.96 \\
\hline \multirow[t]{3}{*}{ Gl 189} & 4.36 & F7 V & 11.65 & $S$ & 0.484500 & 0.109600 & 26.0 & 1236 & 28.67 \\
\hline & & & & $\mathrm{P}$ & 1.459000 & 0.053800 & 4208.0 & 500 & 29.15 \\
\hline & & & & $\mathrm{F}$ & 0.349300 & 0.015200 & 3505.0 & 1507 & 29.23 \\
\hline G1 197 & 4.20 & G2 IV-V & 12.65 & S & $<0.02212$ & & 4.2 & 456 & 27.40 \\
\hline \multirow[t]{2}{*}{ G1 211} & 5.79 & $\mathrm{~K} 1 \mathrm{~V} \mathrm{e}$ & 12.24 & $\mathrm{~S}$ & 0.368900 & 0.032980 & 331.0 & 367 & 28.60 \\
\hline & & & & $\mathrm{P}$ & 0.307700 & 0.007600 & 4435.0 & 5498 & 28.52 \\
\hline \multirow[t]{2}{*}{ Gl 216A } & 3.82 & F6 V & 8.97 & S & 0.343000 & 0.028840 & 330.0 & 488 & 28.30 \\
\hline & & & & $\mathrm{P}$ & 0.032120 & 0.004440 & 64.0 & 2016 & 27.27 \\
\hline \multirow[t]{3}{*}{ G1 222AB } & 4.74 & G0 V & 8.66 & $\mathrm{~S}$ & 1.942000 & 0.068150 & 2940.0 & 431 & 29.02 \\
\hline & & & & $\mathrm{F}$ & 0.408600 & 0.008850 & 9999.0 & 5284 & 29.04 \\
\hline & & & & $\mathrm{H}$ & 0.426400 & 0.011600 & 31.9 & 3119 & 28.96 \\
\hline \multirow[t]{2}{*}{ G1 231} & 5.05 & G5 V & 10.15 & $\mathrm{~S}$ & 0.017000 & 0.004695 & 21.0 & 1166 & 27.10 \\
\hline & & & & $\mathrm{P}$ & 0.024660 & 0.003440 & 50.0 & 2660 & 27.26 \\
\hline \multirow[t]{3}{*}{ Gl 280A } & 2.63 & F5 IV-V & 3.50 & S & 3.655000 & 0.097970 & 5080.0 & 400 & 28.51 \\
\hline & & & & $\mathrm{P}$ & 2.641000 & 0.027000 & 9999.0 & 3654 & 28.37 \\
\hline & & & & $\mathrm{F}$ & 0.360200 & 0.018800 & 3793.0 & 1061 & 28.20 \\
\hline
\end{tabular}


Table 1. continued.

\begin{tabular}{|c|c|c|c|c|c|c|c|c|c|}
\hline $\begin{array}{l}\text { Catalogue } \\
\text { name }\end{array}$ & $\overline{M_{V}}$ & $\begin{array}{l}\text { Spectral } \\
\text { type }\end{array}$ & $\begin{array}{r}\text { Distance } \\
(\mathrm{pc})\end{array}$ & Mode & Count rate & Error & $\begin{array}{r}\text { Likelihood } \\
\text { or } \mathrm{S} / \mathrm{N}\end{array}$ & $\begin{array}{r}\text { Exposure } \\
\text { time }\end{array}$ & $\log L_{X}$ \\
\hline G1 302 & 5.45 & G7.5 V & 12.58 & $S$ & 0.032240 & 0.011320 & 13.0 & 355 & 27.56 \\
\hline Gl 324A & 5.46 & G8 V & 12.53 & S & $<0.04913$ & & 3.6 & 337 & 27.74 \\
\hline Gl 354A & 2.53 & F6 IV & 13.49 & S & $<0.01938$ & & 2.1 & 397 & 27.40 \\
\hline \multirow[t]{3}{*}{ Gl 356A } & 5.17 & G8 V & 11.18 & $S$ & 0.298200 & 0.026960 & 254.0 & 467 & 28.43 \\
\hline & & & & $\mathrm{P}$ & 0.362400 & & 779.0 & 3899 & 28.51 \\
\hline & & & & $\mathrm{H}$ & 0.073430 & 0.004580 & 11.0 & 3526 & 28.42 \\
\hline Gl 395 & 4.29 & F8 V & 12.85 & S & 0.195400 & 0.020980 & 172.0 & 572 & 28.36 \\
\hline Gl $423 \mathrm{AB}$ & 4.72 & G0 V e & 8.35 & S & 4.539000 & 0.157500 & 3380.0 & 192 & 29.36 \\
\hline \multirow[t]{3}{*}{ Gl 434} & 5.43 & G8 V e & 9.54 & S & 0.348000 & 0.037120 & 194.0 & 314 & 28.36 \\
\hline & & & & $\mathrm{P}$ & 0.390100 & 0.014200 & 3272.0 & 1956 & 28.41 \\
\hline & & & & F & 0.090090 & 0.004100 & 3880.0 & 5572 & 28.47 \\
\hline Gl 442A & 5.05 & G5 V & 9.24 & $\mathrm{H}$ & 0.002430 & 0.000621 & 3.6 & 10109 & 26.78 \\
\hline \multirow[t]{2}{*}{ Gl 449} & 3.41 & F9 V & 10.90 & S & 0.343700 & 0.030270 & 264.0 & 417 & 28.47 \\
\hline & & & & $\mathrm{P}$ & 0.377500 & 0.007230 & 4123.0 & 7433 & 28.51 \\
\hline Gl 454 & 5.00 & K0 IV & 12.91 & S & $<0.05198$ & & 7.9 & 352 & 27.79 \\
\hline \multirow[t]{2}{*}{ G1 475} & 4.66 & G0 V & 8.37 & S & 0.023470 & 0.008381 & 12.0 & 509 & 27.07 \\
\hline & & & & $\mathrm{P}$ & 0.036830 & & 148.0 & 20537 & 27.27 \\
\hline Gl 482AB & 3.10 & F0 V & 11.83 & S & 2.039000 & 0.121100 & 888.0 & 146 & 29.31 \\
\hline \multirow[t]{2}{*}{ Gl 502} & 4.46 & G0 V & 9.15 & S & 0.255000 & 0.037070 & 78.0 & 504 & 28.19 \\
\hline & & & & $\mathrm{P}$ & 0.294400 & 0.006210 & 3909.0 & 8010 & 28.25 \\
\hline \multirow[t]{2}{*}{ Gl 506} & 5.08 & G6 V & 8.53 & $\mathrm{P}$ & 0.014160 & 0.002460 & 50.0 & 3113 & 26.87 \\
\hline & & & & $\mathrm{H}$ & 0.002125 & 0.000856 & 2.8 & 5419 & 26.65 \\
\hline \multirow[t]{2}{*}{ G1 534} & 2.41 & G0 IV & 11.34 & S & 0.143800 & 0.023100 & 55.0 & 377 & 28.12 \\
\hline & & & & $\mathrm{P}$ & 0.185100 & 0.005610 & 1770.0 & 6404 & 28.23 \\
\hline \multirow[t]{3}{*}{ Gl 559A } & 4.38 & G2 V & 1.34 & S & 3.876000 & 0.211800 & 589.0 & 420 & 27.70 \\
\hline & & & & $\mathrm{P}$ & 3.357000 & 0.033600 & 9999.0 & 3260 & 27.64 \\
\hline & & & & $\mathrm{H}$ & 0.658200 & 0.014700 & 25.0 & 3038 & 27.53 \\
\hline \multirow[t]{3}{*}{ Gl 559B } & 5.72 & K0 V & 1.34 & $S$ & 3.876000 & 0.211800 & 589.0 & 420 & 27.70 \\
\hline & & & & $\mathrm{P}$ & 3.357000 & 0.033600 & 9999.0 & 3260 & 27.64 \\
\hline & & & & $\mathrm{H}$ & 0.773600 & 0.015900 & 48.9 & 3038 & 27.60 \\
\hline \multirow[t]{4}{*}{ Gl 566A } & 5.59 & G8 V e & 6.70 & S & 2.440000 & 0.183400 & 416.0 & 400 & 28.90 \\
\hline & & & & $\mathrm{P}$ & 2.351000 & 0.020700 & 9999.0 & 5213 & 28.88 \\
\hline & & & & $\mathrm{F}$ & 0.604100 & 0.011900 & 9999.0 & 4270 & 28.99 \\
\hline & & & & $\mathrm{H}$ & 0.767300 & 0.017400 & 32.7 & 2495 & 29.00 \\
\hline \multirow[t]{2}{*}{ G1 567} & 5.70 & K2 V & 11.54 & $S$ & 0.362900 & 0.034130 & 245.0 & 398 & 28.54 \\
\hline & & & & $\mathrm{P}$ & 0.211600 & & 1735.0 & 13998 & 28.31 \\
\hline \multirow[t]{3}{*}{ Gl 575A } & 4.71 & F9 V n & 12.76 & S & 3.414000 & 0.076010 & 9070.0 & 612 & 29.60 \\
\hline & & & & $\mathrm{P}$ & 3.510000 & 0.040400 & 9999.0 & 2166 & 29.61 \\
\hline & & & & $\mathrm{H}$ & 1.141000 & 0.005250 & 190.6 & 40853 & 29.73 \\
\hline \multirow[t]{3}{*}{ Gl 575B } & 5.34 & $\mathrm{dG} 2$ & 12.76 & S & 3.414000 & 0.076010 & 9070.0 & 612 & 29.60 \\
\hline & & & & $\mathrm{P}$ & 3.510000 & 0.040400 & 9999.0 & 2166 & 29.61 \\
\hline & & & & $\mathrm{H}$ & 1.141000 & 0.005250 & 190.6 & 40853 & 29.73 \\
\hline \multirow[t]{2}{*}{ G1 598} & 4.07 & G0 V & 11.75 & $S$ & 0.062080 & 0.014010 & 30.0 & 503 & 27.79 \\
\hline & & & & $\mathrm{P}$ & 0.072190 & 0.005470 & 251.0 & 2726 & 27.85 \\
\hline Gl 601A & 2.40 & F2 IV & 12.31 & S & 0.144300 & 0.024700 & 58.0 & 365 & 28.20 \\
\hline Gl 603 & 3.62 & F6 V & 11.12 & S & 0.035450 & 0.011590 & 10.0 & 560 & 27.50 \\
\hline Gl $620.1 \mathrm{~A}$ & 4.83 & $\mathrm{G} 3 / 5 \mathrm{~V}$ & 12.87 & S & 0.654400 & 0.044910 & 646.0 & 349 & 28.89 \\
\hline & & & & $\mathrm{P}$ & 0.699200 & 0.020600 & 3231.0 & 1724 & 28.92 \\
\hline & & & & $\mathrm{F}$ & 0.181900 & 0.007560 & 4302.0 & 3269 & 29.03 \\
\hline Gl 624 & 4.48 & G0 V & 12.11 & S & 1.027000 & 0.080740 & 493.0 & 166 & 29.03 \\
\hline Gl 635AB & 2.74 & G0 IV & 10.80 & $S$ & 0.127800 & 0.018560 & 77.0 & 494 & 28.03 \\
\hline & & & & $\mathrm{P}$ & 0.128600 & 0.004240 & 2371.0 & 7752 & 28.03 \\
\hline & & & & $\mathrm{H}$ & 0.015160 & 0.003790 & 3.9 & 1219 & 27.71 \\
\hline Gl 666A & 5.75 & G8 V & 8.79 & S & 0.029000 & 0.013510 & 8.0 & 251 & 27.21 \\
\hline & & & & $\mathrm{P}$ & 0.012090 & 0.003340 & 10.0 & 1250 & 26.83 \\
\hline Gl 695Aa & 3.80 & G5 IV & 8.40 & S & 0.197000 & 0.018860 & 232.0 & 718 & 28.00 \\
\hline & & & & $\mathrm{P}$ & 0.163900 & 0.017000 & 95.0 & 587 & 27.92 \\
\hline & & & & $\mathrm{F}$ & 0.025270 & 0.003300 & 98.0 & 2473 & 27.81 \\
\hline & & & & $\mathrm{H}$ & 0.015870 & 0.004320 & 3.6 & 910 & 27.51 \\
\hline
\end{tabular}


Table 1. continued.

\begin{tabular}{|c|c|c|c|c|c|c|c|c|c|}
\hline $\begin{array}{l}\text { Catalogue } \\
\text { name }\end{array}$ & $\overline{M_{V}}$ & $\begin{array}{l}\text { Spectral } \\
\text { type }\end{array}$ & $\begin{array}{r}\text { Distance } \\
(\mathrm{pc}) \\
\end{array}$ & Mode & Count rate & Error & $\begin{array}{r}\text { Likelihood } \\
\text { or } \mathrm{S} / \mathrm{N} \\
\end{array}$ & $\begin{array}{r}\text { Exposure } \\
\text { time } \\
\end{array}$ & $\log L_{X}$ \\
\hline \multirow[t]{3}{*}{ Gl 702A } & 5.68 & K0 V e & 5.09 & $\mathrm{~S}$ & 1.649000 & 0.127200 & 410.0 & 347 & 28.49 \\
\hline & & & & $\mathrm{F}$ & 0.247300 & 0.011500 & 4242.0 & 1903 & 28.36 \\
\hline & & & & $\mathrm{H}$ & 0.210900 & 0.016400 & 11.0 & 778 & 28.20 \\
\hline \multirow[t]{2}{*}{ Gl 713A } & 4.15 & F7 V & 8.06 & $\mathrm{~S}$ & 0.155800 & 0.009018 & 506.0 & 2629 & 27.86 \\
\hline & & & & $\mathrm{P}$ & 0.152800 & 0.006840 & 2255.0 & 3490 & 27.85 \\
\hline Gl 722 & 5.26 & G5 V & 12.98 & $\mathrm{~S}$ & 0.045080 & 0.016000 & 15.0 & 283 & 27.74 \\
\hline \multirow[t]{2}{*}{ Gl 771A } & 3.04 & G8 IV & 13.71 & $\mathrm{~S}$ & 0.247900 & 0.029290 & 202.0 & 343 & 28.52 \\
\hline & & & & $\mathrm{H}$ & 0.052870 & 0.006060 & 7.6 & 1474 & 28.46 \\
\hline \multirow[t]{2}{*}{ Gl 780} & 4.62 & G8 V & 6.11 & $S$ & 0.073070 & 0.027780 & 10.0 & 124 & 27.29 \\
\hline & & & & $\mathrm{H}$ & 0.018620 & 0.002170 & 8.2 & 4350 & 27.30 \\
\hline \multirow[t]{2}{*}{ Gl 827} & 4.38 & F8 V & 9.22 & $\mathrm{P}$ & 0.009295 & 0.002730 & 22.0 & 1820 & 26.75 \\
\hline & & & & $\mathrm{H}$ & 0.004878 & 0.001060 & 4.9 & 5914 & 27.08 \\
\hline Gl 848AB & 3.41 & F5 V & 11.76 & $S$ & 0.259900 & 0.024040 & 261.0 & 505 & 28.41 \\
\hline Gl 853A & 4.71 & G1 V & 13.61 & $\mathrm{~S}$ & 0.040330 & 0.013370 & 12.0 & 359 & 27.73 \\
\hline \multirow[t]{2}{*}{ G1 903} & 2.51 & K1 IVe & 13.79 & S & 0.014180 & 0.004882 & 12.0 & 977 & 27.29 \\
\hline & & & & $\mathrm{P}$ & 0.015300 & 0.001930 & 110.0 & 5669 & 27.32 \\
\hline Gl 904 & 3.42 & F7 V & 13.79 & S & 0.111500 & 0.019300 & 73.0 & 373 & 28.18 \\
\hline Gl 914A & 5.34 & G3 V & 12.40 & S & 0.029120 & 0.011240 & 12.0 & 369 & 27.51 \\
\hline
\end{tabular}

Table 2. Sample stars with absolute magnitude between 5.80 and 8.50 and a distance up to 12 pc, the K-type stars; columns as in Table 1 .

\begin{tabular}{|c|c|c|c|c|c|c|c|c|c|}
\hline $\begin{array}{l}\text { Catalogue } \\
\text { name }\end{array}$ & $M_{V}$ & $\begin{array}{l}\text { Spectral } \\
\text { type }\end{array}$ & $\begin{array}{r}\text { Distance } \\
(\mathrm{pc})\end{array}$ & Mode & Count rate & Error & $\begin{array}{r}\text { Likelihood } \\
\text { or } \mathrm{S} / \mathrm{N}\end{array}$ & $\begin{array}{r}\text { Exposure } \\
\text { time }\end{array}$ & $\log L_{X}$ \\
\hline Gl 33 & 6.37 & $\mathrm{~K} 2 \mathrm{~V}$ & 7.46 & $\mathrm{~S}$ & 0.043170 & 0.013090 & 17.0 & 353 & 27.24 \\
\hline Gl 66AB & 6.26 & $\mathrm{~K} 2 \mathrm{~V}$ & 8.15 & S & 0.260478 & 0.039944 & 99.0 & 189 & 29.09 \\
\hline \multirow[t]{2}{*}{ Gl 68} & 5.87 & $\mathrm{~K} 1 \mathrm{~V}$ & 7.47 & $\mathrm{P}$ & 0.026610 & 0.002680 & 135.0 & 4076 & 27.03 \\
\hline & & & & $\mathrm{H}$ & 0.008686 & 0.001860 & 4.4 & 3055 & 27.14 \\
\hline \multirow[t]{2}{*}{ Gl 86} & 5.92 & K0 V & 10.91 & $\mathrm{~S}$ & 0.058230 & 0.015840 & 24.0 & 375 & 27.70 \\
\hline & & & & $\mathrm{P}$ & 0.106900 & 0.005600 & 1046.0 & 3587 & 27.96 \\
\hline G1 92B & 6.78 & & 10.85 & S & 0.423400 & 0.039160 & 285.0 & 299 & 28.55 \\
\hline \multirow[t]{2}{*}{ Gl 105A } & 6.53 & K3 V & 7.21 & $S$ & 0.058490 & 0.019040 & 19.0 & 232 & 27.34 \\
\hline & & & & $\mathrm{H}$ & 0.016400 & 0.000909 & 16.4 & 21213 & 27.39 \\
\hline \multirow[t]{3}{*}{ Gl 117} & 5.96 & $\mathrm{~K} 2 \mathrm{~V}$ & 10.38 & $\mathrm{~S}$ & 1.200000 & 0.077170 & 883.0 & 220 & 28.97 \\
\hline & & & & $\mathrm{P}$ & 1.081000 & 0.016700 & 9999.0 & 3880 & 28.92 \\
\hline & & & & $\mathrm{F}$ & 0.314700 & 0.006020 & 9999.0 & 8609 & 29.09 \\
\hline \multirow[t]{3}{*}{ Gl 144} & 6.18 & $\mathrm{~K} 2 \mathrm{~V}$ & 3.22 & S & 2.822000 & 0.233500 & 327.0 & 374 & 28.32 \\
\hline & & & & $\mathrm{F}$ & 0.532700 & 0.013400 & 9999.0 & 3045 & 28.30 \\
\hline & & & & $\mathrm{H}$ & 0.712300 & 0.012400 & 51.8 & 4585 & 28.33 \\
\hline \multirow[t]{2}{*}{ Gl 166A } & 5.91 & $\mathrm{~K} 1 \mathrm{~V} \mathrm{e}$ & 5.04 & $S$ & 0.179500 & 0.024990 & 93.0 & 341 & 27.52 \\
\hline & & & & $\mathrm{H}$ & 0.020360 & 0.004740 & 4.2 & 1022 & 27.17 \\
\hline Gl 169 & 7.99 & K7 V & 11.47 & $\mathrm{~S}$ & 0.133200 & 0.019570 & 116.0 & 429 & 28.10 \\
\hline Gl 183 & 6.50 & $\mathrm{~K} 3 \mathrm{~V}$ & 8.81 & $S$ & 0.032200 & 0.012500 & 9.0 & 387 & 27.25 \\
\hline \multirow[t]{3}{*}{ Gl 216B } & 6.41 & $\mathrm{~K} 2 \mathrm{~V}$ & 8.97 & S & 0.343000 & 0.028840 & 330.0 & 488 & 28.30 \\
\hline & & & & $\mathrm{P}$ & 0.273300 & 0.011900 & 2705.0 & 2013 & 28.20 \\
\hline & & & & $\mathrm{F}$ & 0.053350 & 0.003930 & 2350.0 & 3540 & 28.19 \\
\hline \multirow[t]{2}{*}{ Gl 250A } & 6.89 & K3 V & 8.70 & $S$ & 0.229300 & 0.031610 & 152.0 & 269 & 28.10 \\
\hline & & & & $\mathrm{H}$ & 0.029110 & 0.002950 & 9.0 & 3481 & 27.80 \\
\hline \multirow[t]{3}{*}{ Gl 320} & 6.32 & $\mathrm{~K} 1 \mathrm{~V}$ & 11.14 & $S$ & 0.460300 & 0.046610 & 216.0 & 505 & 28.61 \\
\hline & & & & $\mathrm{P}$ & 0.475400 & 0.023800 & 1128.0 & 921 & 28.63 \\
\hline & & & & F & 0.144100 & 0.006420 & 3700.0 & 3562 & 28.81 \\
\hline Gl 325A & 8.43 & K5 V & 11.43 & $\mathrm{~S}$ & 0.059010 & 0.014350 & 25.0 & 423 & 27.74 \\
\hline Gl 370 & 7.43 & K5 V & 11.15 & $\mathrm{H}$ & 0.006027 & 0.001050 & 5.2 & 7168 & 27.33 \\
\hline \multirow[t]{2}{*}{ Gl 380} & 8.15 & $\mathrm{~K} 7 \mathrm{~V}$ & 4.87 & $\mathrm{~S}$ & 0.176800 & 0.021330 & 153.0 & 494 & 27.48 \\
\hline & & & & $\mathrm{P}$ & 0.160100 & & 584.0 & 3296 & 27.44 \\
\hline \multirow[t]{2}{*}{ Gl 414A } & 7.96 & K8 V & 11.93 & $\mathrm{~S}$ & 0.046070 & 0.015680 & 10.0 & 317 & 27.67 \\
\hline & & & & $\mathrm{H}$ & 0.006124 & 0.001510 & 4.6 & 3517 & 27.40 \\
\hline
\end{tabular}


Table 2. continued.

\begin{tabular}{|c|c|c|c|c|c|c|c|c|c|}
\hline $\begin{array}{l}\text { Catalogue } \\
\text { name }\end{array}$ & $M_{V}$ & $\begin{array}{l}\text { Spectral } \\
\text { type }\end{array}$ & $\begin{array}{r}\text { Distance } \\
(\mathrm{pc})\end{array}$ & Mode & Count rate & Error & $\begin{array}{r}\text { Likelihood } \\
\text { or } \mathrm{S} / \mathrm{N}\end{array}$ & $\begin{array}{r}\text { Exposure } \\
\text { time }\end{array}$ & $\log L_{\mathrm{X}}$ \\
\hline Gl 432A & 6.06 & K0 V & 9.54 & $\mathrm{H}$ & 0.003167 & 0.000877 & 3.8 & 6478 & 26.92 \\
\hline Gl 451A & 6.64 & G8 VI & 9.16 & $\mathrm{P}$ & 0.005465 & 0.001690 & 8.0 & 2765 & 26.52 \\
\hline Gl 453 & 6.94 & K5 V & 10.19 & $\mathrm{H}$ & 0.014320 & 0.001290 & 11.2 & 9483 & 27.63 \\
\hline Gl 488 & 8.33 & $\mathrm{M} 0.5 \mathrm{Ve}$ & 10.78 & $\mathrm{~S}$ & 0.061280 & 0.020860 & 12.0 & 221 & 27.71 \\
\hline \multirow[t]{3}{*}{ Gl 505A } & 6.34 & K1 V & 11.23 & $S$ & 0.142800 & 0.026870 & 48.0 & 279 & 28.11 \\
\hline & & & & $\mathrm{P}$ & 0.209100 & 0.011400 & 1338.0 & 1656 & 28.28 \\
\hline & & & & $\mathrm{F}$ & 0.040800 & 0.003290 & 315.0 & 3634 & 28.27 \\
\hline Gl 542 & 6.29 & K3 V & 11.83 & $\mathrm{H}$ & 0.009136 & 0.002100 & 3.2 & 2445 & 27.56 \\
\hline \multirow[t]{4}{*}{ Gl 566B } & 7.82 & $\mathrm{~K} 4 \mathrm{~V}$ e & 6.70 & $\mathrm{~S}$ & 2.440000 & 0.183400 & 416.0 & 400 & 28.90 \\
\hline & & & & $\mathrm{P}$ & 2.351000 & 0.020700 & 9999.0 & 5213 & 28.88 \\
\hline & & & & $\mathrm{F}$ & 0.604100 & 0.011900 & 9999.0 & 4270 & 28.99 \\
\hline & & & & $\mathrm{H}$ & 0.767300 & 0.017400 & 32.7 & 2495 & 29.00 \\
\hline \multirow[t]{3}{*}{ Gl 570A } & 6.90 & $\mathrm{~K} 5 \mathrm{Ve}$ & 5.90 & $\mathrm{~S}$ & 0.173100 & 0.070810 & 9.0 & 44 & 27.64 \\
\hline & & & & $\mathrm{P}$ & 0.238000 & & 1305.0 & 5321 & 27.77 \\
\hline & & & & $\mathrm{H}$ & 0.040760 & 0.004130 & 9.4 & 2443 & 27.61 \\
\hline \multirow[t]{2}{*}{ Gl 617A } & 8.45 & M0 V e & 10.69 & S & 0.059910 & 0.007541 & 91.0 & 1726 & 27.69 \\
\hline & & & & $\mathrm{H}$ & 0.009817 & 0.001580 & 5.9 & 4503 & 27.51 \\
\hline Gl 631 & 5.80 & K0 V e & 9.78 & $S$ & 0.257900 & 0.031920 & 148.0 & 603 & 28.25 \\
\hline Gl 638 & 8.16 & K7 V & 9.77 & $S$ & 0.026230 & 0.009423 & 11.0 & 468 & 27.25 \\
\hline Gl 653 & 7.57 & K7 V & 10.76 & $\mathrm{H}$ & 0.002910 & & $7.4^{1}$ & 2213 & 26.99 \\
\hline \multirow[t]{4}{*}{ Gl $663 \mathrm{AB}$} & 6.16 & $\mathrm{~K} 1 \mathrm{~V}$ e & 5.99 & $\mathrm{~S}$ & 0.854100 & 0.057780 & 664.0 & 291 & 28.34 \\
\hline & & & & $\mathrm{P}$ & 1.171000 & 0.025700 & 9999.0 & 1783 & 28.48 \\
\hline & & & & $\mathrm{F}$ & 0.265100 & 0.013900 & 3394.0 & 1429 & 28.53 \\
\hline & & & & $\mathrm{H}$ & 0.146100 & 0.008600 & 14.2 & 1973 & 28.18 \\
\hline \multirow[t]{4}{*}{ Gl 664} & 7.46 & $\mathrm{~K} 5 \mathrm{~V} \mathrm{e}$ & 5.97 & $\mathrm{~S}$ & 0.254600 & 0.031500 & 157.0 & 288 & 27.81 \\
\hline & & & & $\mathrm{P}$ & 0.265000 & 0.012800 & 2360.0 & 1665 & 27.83 \\
\hline & & & & $\mathrm{F}$ & 0.050210 & 0.006150 & 165.0 & 1391 & 27.81 \\
\hline & & & & $\mathrm{H}$ & 0.043430 & 0.005410 & 4.9 & 1972 & 27.65 \\
\hline \multirow[t]{2}{*}{ Gl 667AB } & 6.99 & K3 V & 7.25 & $\mathrm{~S}$ & 0.205700 & 0.028810 & 120.0 & 307 & 27.89 \\
\hline & & & & $\mathrm{H}$ & 0.035800 & 0.004370 & 7.7 & 1916 & 27.73 \\
\hline Gl 673 & 8.10 & K7 V & 7.72 & $\mathrm{~S}$ & 0.090570 & 0.018050 & 40.0 & 445 & 27.59 \\
\hline Gl 688 & 6.37 & K3 V & 10.71 & $S$ & 0.048270 & 0.013440 & 19.0 & 440 & 27.60 \\
\hline \multirow[t]{3}{*}{ Gl 702B } & 7.48 & $\mathrm{~K} 5 \mathrm{~V} \mathrm{e}$ & 5.09 & S & 1.649000 & 0.127200 & 410.0 & 347 & 28.49 \\
\hline & & & & F & 0.247300 & 0.011500 & 4242.0 & 1903 & 28.36 \\
\hline & & & & $\mathrm{H}$ & 0.210900 & 0.016400 & 11.0 & 778 & 28.20 \\
\hline Gl 706 & 6.17 & K2 V & 11.10 & $\mathrm{H}$ & 0.002579 & 0.000815 & 4.0 & 6294 & 26.96 \\
\hline \multirow[t]{2}{*}{ Gl 713B } & 6.59 & & 8.06 & $\mathrm{~S}$ & 0.155800 & 0.009018 & 506.0 & 2629 & 27.86 \\
\hline & & & & $\mathrm{P}$ & 0.152800 & 0.006840 & 2255.0 & 3490 & 27.85 \\
\hline \multirow[t]{3}{*}{ G1 764} & 5.88 & K0 V & 5.77 & $S$ & 0.255700 & 0.014290 & 694.0 & 1448 & 27.79 \\
\hline & & & & $\mathrm{P}$ & 0.171300 & 0.008160 & 2348.0 & 2684 & 27.61 \\
\hline & & & & $\mathrm{H}$ & 0.036140 & 0.006330 & 4.9 & 923 & 27.54 \\
\hline Gl 773.6 & 8.35 & K5 V & 11.80 & $\mathrm{~S}$ & $<0.02880$ & & 1.5 & 307 & 27.45 \\
\hline \multirow[t]{2}{*}{ Gl 783A } & 6.41 & K3 V & 6.05 & $\mathrm{P}$ & 0.027630 & 0.004230 & 72.0 & 1883 & 26.86 \\
\hline & & & & $\mathrm{H}$ & 0.006246 & 0.001100 & 5.4 & 6530 & 26.82 \\
\hline Gl 785 & 6.00 & K0 V & 8.82 & $\mathrm{~S}$ & 0.061820 & 0.026730 & 10.0 & 126 & 27.54 \\
\hline \multirow[t]{3}{*}{ Gl 820A } & 7.50 & K5 V & 3.50 & $S$ & 0.421700 & 0.030030 & 452.0 & 543 & 27.57 \\
\hline & & & & F & 0.057470 & 0.003430 & 1282.0 & 4934 & 27.40 \\
\hline & & & & $\mathrm{H}$ & 0.071220 & 0.003280 & 24.2 & 6688 & 27.40 \\
\hline \multirow[t]{3}{*}{ Gl 820B } & 8.32 & K7 V & 3.50 & $\mathrm{~S}$ & 0.421700 & 0.030030 & 452.0 & 543 & 27.57 \\
\hline & & & & $\mathrm{F}$ & 0.057470 & 0.003430 & 1282.0 & 4934 & 27.40 \\
\hline & & & & $\mathrm{H}$ & 0.024390 & 0.001960 & 11.4 & 6688 & 26.93 \\
\hline \multirow[t]{4}{*}{ Gl 845} & 6.89 & K5 V e & 3.63 & $\mathrm{~S}$ & 0.260400 & 0.028540 & 169.0 & 414 & 27.39 \\
\hline & & & & $\mathrm{P}$ & 0.507900 & 0.006080 & 9999.0 & 14198 & 27.68 \\
\hline & & & & F & 0.081000 & 0.004290 & 2792.0 & 4410 & 27.58 \\
\hline & & & & $\mathrm{H}$ & 0.057770 & 0.005510 & 9.3 & 1958 & 27.34 \\
\hline Gl 879 & 7.07 & $\mathrm{~K} 5 \mathrm{~V} \mathrm{e}$ & 7.64 & $\mathrm{~S}$ & 0.526300 & 0.065160 & 185.0 & 150 & 28.34 \\
\hline Gl 884 & 8.34 & K5 V & 8.14 & S & $<0.11527$ & & 6.3 & 49 & 27.39 \\
\hline Gl 892 & 6.50 & K3 V & 6.53 & S & 0.023110 & 0.008761 & 10.0 & 497 & 26.85 \\
\hline Gl 902 & 6.81 & K3 V & 11.40 & $S$ & 0.029190 & 0.010000 & 12.0 & 462 & 27.44 \\
\hline
\end{tabular}


Table 2. continued.

\begin{tabular}{lclrcccrrr}
\hline \hline $\begin{array}{l}\text { Catalogue } \\
\text { name }\end{array}$ & $M_{V}$ & $\begin{array}{l}\text { Spectral } \\
\text { type }\end{array}$ & $\begin{array}{r}\text { Distance } \\
(\mathrm{pc})\end{array}$ & Mode & Count rate & Error & $\begin{array}{r}\text { Likelihood } \\
\text { or S/N }\end{array}$ & $\begin{array}{r}\text { Exposure } \\
\text { time }\end{array}$ & $\log L_{X}$ \\
\hline G1 909A & 6.24 & $\mathrm{~K} 3 \mathrm{~V}$ & 10.79 & $\mathrm{~S}$ & 0.419100 & 0.024660 & 795.0 & 747 & 28.54 \\
LTT 14084 & 6.50 & $\mathrm{~K} 3 \mathrm{~V}$ & 10.10 & $\mathrm{~S}$ & 0.016520 & 0.006606 & 9.0 & 813 & 27.08 \\
\hline
\end{tabular}

Table 3. Sample stars with absolute magnitude fainter than 8.50 and a distance up to 6 pc, M-type stars; columns as in Table 1 .

\begin{tabular}{|c|c|c|c|c|c|c|c|c|c|}
\hline $\begin{array}{l}\text { Catalogue } \\
\text { name }\end{array}$ & $M_{V}$ & $\begin{array}{l}\text { Spectral } \\
\text { type }\end{array}$ & $\begin{array}{r}\text { Distance } \\
(\mathrm{pc})\end{array}$ & Mode & Count rate & Error & $\begin{array}{r}\text { Likelihood } \\
\text { or } \mathrm{S} / \mathrm{N}\end{array}$ & $\begin{array}{r}\text { Exposure } \\
\text { time }\end{array}$ & $\log L_{X}$ \\
\hline \multirow[t]{2}{*}{ Gl 1} & 10.35 & M3 V & 4.36 & $\bar{S}$ & 0.059340 & 0.024300 & 9.0 & 156 & 26.91 \\
\hline & & & & $\mathrm{P}$ & 0.019690 & 0.002380 & 104.0 & 4604 & 26.43 \\
\hline \multirow[t]{2}{*}{ Gl 15A } & 10.32 & M1.5 V & 3.57 & S & 0.260200 & 0.026180 & 202.0 & 453 & 27.38 \\
\hline & & & & $\mathrm{H}$ & 0.013900 & 0.003060 & 4.4 & 1606 & 26.71 \\
\hline \multirow[t]{2}{*}{ Gl 15B } & 13.29 & M3.5 V & 3.57 & $S$ & 0.260200 & 0.026180 & 202.0 & 453 & 27.38 \\
\hline & & & & $\mathrm{H}$ & 0.053440 & 0.005780 & 8.7 & 1606 & 27.29 \\
\hline \multirow[t]{2}{*}{ Gl 34B } & 8.64 & K7 V & 5.95 & $S$ & 0.137900 & 0.021990 & 71.0 & 381 & 27.54 \\
\hline & & & & $\mathrm{H}$ & 0.026470 & 0.002070 & 10.2 & 6391 & 27.43 \\
\hline \multirow[t]{2}{*}{ Gl 54.1} & 14.19 & M4.5 V & 3.72 & S & 0.253800 & 0.025840 & 200.0 & 461 & 27.40 \\
\hline & & & & $\mathrm{P}$ & 0.079810 & 0.008050 & 142.0 & 1359 & 26.90 \\
\hline \multirow[t]{3}{*}{ Gl 65AB } & 15.41 & M5.5 V & 2.68 & S & 0.680300 & 0.056820 & 318.0 & 484 & 27.55 \\
\hline & & & & $\mathrm{P}$ & 0.355700 & & 3253.0 & 16136 & 27.26 \\
\hline & & & & $\mathrm{H}$ & 1.410000 & 0.010100 & 121.7 & 13884 & 28.46 \\
\hline G1 83.1 & 14.03 & M4.5 V & 4.45 & S & 0.167400 & 0.024950 & 111.0 & 295 & 27.38 \\
\hline \multirow[t]{2}{*}{ G1 166C } & 12.69 & M4.5 V & 5.04 & $S$ & 0.796400 & 0.051660 & 572.0 & 344 & 28.16 \\
\hline & & & & $\mathrm{H}$ & 0.107100 & 0.010400 & 9.8 & 1022 & 27.89 \\
\hline Gl $169.1 \mathrm{~A}$ & 12.34 & M4 V & 5.51 & $\mathrm{P}$ & 0.011920 & 0.002220 & 41.0 & 3323 & 26.41 \\
\hline \multirow[t]{2}{*}{ Gl 191} & 10.88 & M1 p V & 3.92 & S & 0.048740 & 0.019720 & 10.0 & 248 & 26.73 \\
\hline & & & & $\mathrm{P}$ & 0.018180 & 0.001320 & 353.0 & 14268 & 26.30 \\
\hline \multirow[t]{2}{*}{ G1 205} & 9.19 & M1.5 V & 5.69 & S & 0.196300 & 0.022530 & 152.0 & 455 & 27.66 \\
\hline & & & & $\mathrm{P}$ & 0.187000 & 0.006120 & 2014.0 & 5156 & 27.64 \\
\hline \multirow[t]{2}{*}{ G1 213} & 12.70 & M4 V & 5.79 & $S$ & 0.016150 & 0.006921 & 9.0 & 461 & 26.59 \\
\hline & & & & $\mathrm{P}$ & 0.004701 & 0.000729 & 43.0 & 13362 & 26.05 \\
\hline Gl 229AB & 9.34 & M1 V & 5.77 & $S$ & 0.053500 & 0.011580 & 39.0 & 592 & 27.11 \\
\hline Gl 234AB & 13.08 & $\mathrm{M} 4.0 \mathrm{~V}$ & 4.12 & $S$ & 0.767000 & & & 490 & 27.97 \\
\hline Gl 251 & 11.31 & M3 V & 5.52 & $\mathrm{P}$ & 0.005924 & 0.001500 & 30.0 & 4048 & 26.11 \\
\hline \multirow[t]{2}{*}{ Gl 273} & 11.95 & M3.5 V & 3.80 & S & 0.034000 & 0.014910 & 9.0 & 247 & 26.55 \\
\hline & & & & $\mathrm{P}$ & 0.010390 & 0.002310 & 37.0 & 2932 & 26.03 \\
\hline G1 285 & 12.30 & M4 V & 5.93 & $S$ & 1.467000 & 0.078880 & 1220.0 & 253 & 28.57 \\
\hline G1 300 & 13.22 & M3.5 V & 5.89 & $\mathrm{P}$ & 0.007263 & 0.001580 & 28.0 & 4096 & 26.26 \\
\hline \multirow[t]{3}{*}{ Gl 388} & 10.95 & M3 V & 4.89 & $S$ & 3.701000 & 0.194400 & 1500.0 & 104 & 28.80 \\
\hline & & & & $\mathrm{P}$ & 2.226000 & 0.013500 & 9999.0 & 25092 & 28.58 \\
\hline & & & & $\mathrm{H}$ & 0.698700 & 0.006710 & 94.6 & 15302 & 28.68 \\
\hline \multirow[t]{2}{*}{ Gl 406} & 16.56 & M6 V & 2.39 & S & 0.227500 & 0.025720 & 183.0 & 384 & 26.97 \\
\hline & & & & $\mathrm{P}$ & 0.250000 & 0.009960 & 2566.0 & 2604 & 27.01 \\
\hline \multirow[t]{2}{*}{ Gl 411} & 10.46 & M2 V & 2.55 & $S$ & 0.167000 & 0.024170 & 83.0 & 341 & 26.89 \\
\hline & & & & $\mathrm{P}$ & 0.212400 & 0.004760 & 3844.0 & 10006 & 27.00 \\
\hline \multirow[t]{2}{*}{ Gl 412A } & 10.34 & M1 V & 4.83 & S & 0.184800 & 0.026840 & 111.0 & 364 & 27.49 \\
\hline & & & & $\mathrm{H}$ & 0.005208 & 0.000526 & 5.0 & 24751 & 26.54 \\
\hline \multirow[t]{2}{*}{ Gl 412B } & 15.99 & M5.5 V & 4.83 & $\mathrm{~S}$ & 0.184800 & 0.026840 & 111.0 & 364 & 27.49 \\
\hline & & & & $\mathrm{H}$ & 0.042180 & 0.001320 & 25.4 & 24751 & 27.45 \\
\hline Gl 445 & 12.14 & M3.5 V & 5.39 & $\mathrm{P}$ & 0.021170 & 0.004150 & 41.0 & 1485 & 26.65 \\
\hline \multirow[t]{2}{*}{ Gl 447} & 13.50 & M4 V & 3.34 & S & 0.079610 & 0.017270 & 41.0 & 364 & 26.80 \\
\hline & & & & $\mathrm{P}$ & 0.052780 & & 174.0 & 3885 & 26.63 \\
\hline \multirow[t]{2}{*}{ Gl 473AB } & 14.99 & M5.5 V & 4.39 & $S$ & 0.238600 & 0.026880 & 147.0 & 430 & 27.52 \\
\hline & & & & $\mathrm{P}$ & 0.295600 & 0.012900 & 2437.0 & 1922 & 27.61 \\
\hline G1 526 & 9.80 & M1.5 V & 5.43 & $\mathrm{P}$ & 0.035310 & 0.007460 & 36.0 & 747 & 26.87 \\
\hline \multirow[t]{2}{*}{ Gl 551} & 15.48 & M5.5 & 1.29 & S & 1.374000 & 0.110700 & 445.0 & 374 & 27.22 \\
\hline & & & & $\mathrm{P}$ & 0.541100 & 0.005680 & 9999.0 & 17157 & 26.81 \\
\hline
\end{tabular}


Table 3. continued.

\begin{tabular}{|c|c|c|c|c|c|c|c|c|c|}
\hline $\begin{array}{l}\text { Catalogue } \\
\text { name }\end{array}$ & $\overline{M_{V}}$ & $\begin{array}{l}\text { Spectral } \\
\text { type }\end{array}$ & $\begin{array}{r}\text { Distance } \\
(\mathrm{pc}) \\
\end{array}$ & Mode & Count rate & Error & $\begin{array}{r}\text { Likelihood } \\
\text { or } \mathrm{S} / \mathrm{N} \\
\end{array}$ & $\begin{array}{r}\text { Exposure } \\
\text { time }\end{array}$ & $\overline{\log L_{X}}$ \\
\hline & & & & $\bar{F}$ & 0.930500 & 0.035500 & 9999.0 & 725 & 27.74 \\
\hline \multirow[t]{3}{*}{ Gl 570BC } & 9.35 & M1 V & 5.90 & S & 0.173100 & 0.070810 & 9.0 & 44 & 27.64 \\
\hline & & & & $P$ & 0.238000 & & 1305.0 & 5321 & 27.77 \\
\hline & & & & $\mathrm{H}$ & 0.028530 & 0.003470 & 7.4 & 2443 & 27.46 \\
\hline Gl 570D & & T8 & 5.90 & $\mathrm{H}$ & $<0.00158$ & & $1.3^{1}$ & 2430 & 26.20 \\
\hline \multirow[t]{2}{*}{ Gl 588} & 10.44 & $\mathrm{M} 2.5 \mathrm{~V}$ & 5.93 & $S$ & 0.039920 & 0.012640 & 13.0 & 416 & 27.00 \\
\hline & & & & $\mathrm{P}$ & 0.026350 & & 61.0 & 4331 & 26.82 \\
\hline Gl 628 & 11.93 & M3 V & 4.26 & S & 0.047450 & 0.011080 & 27.0 & 554 & 26.79 \\
\hline Gl 674 & 11.10 & M3 V & 4.54 & S & 0.266600 & 0.040960 & 76.0 & 193 & 27.60 \\
\hline Gl 682 & 12.45 & $\mathrm{M} 4.5 \mathrm{~V}$ & 5.04 & $\mathrm{P}$ & 0.007986 & 0.002780 & 8.0 & 1433 & 26.16 \\
\hline \multirow[t]{3}{*}{ Gl $687 \mathrm{AB}$} & 10.88 & M3 V & 4.53 & S & 0.062050 & 0.004069 & 374.0 & 5788 & 26.96 \\
\hline & & & & $\mathrm{P}$ & 0.062300 & 0.003340 & 1259.0 & 6274 & 26.96 \\
\hline & & & & $\mathrm{H}$ & 0.020350 & 0.001200 & 15.3 & 15193 & 27.08 \\
\hline Gl 693 & 11.93 & M3.5 V & 5.81 & $\mathrm{~S}$ & $<0.03522$ & & 0.7 & 129 & 26.93 \\
\hline \multirow[t]{2}{*}{ Gl 699} & 13.25 & M4 V & 1.82 & $S$ & 0.029760 & 0.011060 & 13.0 & 399 & 25.85 \\
\hline & & & & $\mathrm{P}$ & 0.023370 & 0.002620 & 105.0 & 4127 & 25.74 \\
\hline \multirow[t]{3}{*}{ Gl 725AB } & 11.14 & M3 V & 3.56 & S & 0.049080 & 0.006292 & 91.0 & 1781 & 26.65 \\
\hline & & & & $\mathrm{P}$ & 0.062860 & 0.005710 & 158.0 & 2263 & 26.76 \\
\hline & & & & $\mathrm{H}$ & 0.021700 & 0.002330 & 8.3 & 4178 & 26.90 \\
\hline \multirow[t]{2}{*}{ Gl 729} & 13.09 & M3.5 V & 2.97 & $\mathrm{~S}$ & 0.942000 & 0.080930 & 334.0 & 326 & 27.78 \\
\hline & & & & $\mathrm{H}$ & 0.204200 & 0.006880 & 27.5 & 4305 & 27.71 \\
\hline \multirow[t]{2}{*}{ Gl 752A } & 10.28 & M3 V & 5.87 & $\mathrm{P}$ & 0.035360 & & 139.0 & 10492 & 26.94 \\
\hline & & & & $\mathrm{H}$ & 0.011460 & 0.000778 & 14.6 & 21758 & 27.05 \\
\hline Gl 752B & 18.68 & M8 V & 5.87 & $\mathrm{H}$ & 0.000444 & 0.000323 & 3.0 & 21757 & 25.64 \\
\hline Gl 754 & 13.41 & M4.5 & 5.71 & $\mathrm{P}$ & 0.004324 & 0.000974 & 15.0 & 6529 & 26.01 \\
\hline \multirow[t]{2}{*}{ Gl 825} & 8.70 & M0.5 V & 3.95 & S & 0.137300 & 0.022020 & 83.0 & 403 & 27.19 \\
\hline & & & & $\mathrm{P}$ & 0.248600 & 0.005250 & 3589.0 & 9467 & 27.44 \\
\hline Gl 832 & 10.20 & M1 V & 4.94 & $\mathrm{P}$ & 0.033740 & 0.004580 & 54.0 & 2041 & 26.77 \\
\hline Gl 860AB & 11.76 & M3 V & 4.04 & S & 0.448600 & 0.033550 & 468.0 & 427 & 27.72 \\
\hline \multirow[t]{2}{*}{ Gl 866AB } & 15.18 & M5 V & 3.45 & S & 0.196900 & 0.038900 & 57.0 & 161 & 27.23 \\
\hline & & & & $\mathrm{P}$ & 0.269900 & 0.004640 & 3800.0 & 12892 & 27.36 \\
\hline \multirow[t]{2}{*}{ Gl 873} & 11.71 & M3.5 V & 5.05 & $\mathrm{~S}$ & 5.384000 & 0.098370 & 9999.0 & 560 & 28.99 \\
\hline & & & & $\mathrm{P}$ & 0.784500 & & 5458.0 & 4463 & 28.16 \\
\hline Gl 876 & 11.80 & M3.5 V & 4.70 & $\mathrm{P}$ & 0.019320 & 0.003770 & 34.0 & 1766 & 26.49 \\
\hline \multirow[t]{2}{*}{ Gl 887} & 9.76 & M2 V & 3.29 & S & 0.137400 & 0.037470 & 28.0 & 148 & 27.03 \\
\hline & & & & $\mathrm{P}$ & 0.222000 & 0.004350 & 3425.0 & 12729 & 27.24 \\
\hline Gl 905 & 14.79 & M5.5 V & 3.16 & S & 0.177200 & 0.023810 & 100.0 & 350 & 27.10 \\
\hline Gl 908 & 10.11 & M1 V & 5.97 & S & 0.051160 & 0.013450 & 27.0 & 414 & 27.12 \\
\hline GJ 1002 & 15.40 & M5.5 V & 4.69 & $\mathrm{P}$ & $<0.00219$ & & 0.3 & 1673 & 25.54 \\
\hline GJ $1005 \mathrm{AB}$ & 12.99 & M3.5 V & 5.36 & $\mathrm{P}$ & 0.007931 & 0.002540 & 10.0 & 1643 & 26.21 \\
\hline GJ 1061 & 15.17 & M5.5 V & 3.70 & $\mathrm{P}$ & 0.011900 & 0.003690 & 16.0 & 1321 & 26.07 \\
\hline GJ 1111 & 16.99 & M6.5 V & 3.63 & $\mathrm{P}$ & 0.039730 & & 264.0 & 10312 & 26.58 \\
\hline \multirow[t]{3}{*}{ GJ 1116AB } & 15.48 & M5.5 V & 5.23 & S & 0.340000 & 0.034000 & 231.0 & 337 & 27.82 \\
\hline & & & & $\mathrm{P}$ & 0.171700 & & 389.0 & 3021 & 27.53 \\
\hline & & & & $\mathrm{H}$ & 0.070920 & 0.003670 & 17.9 & 5319 & 27.75 \\
\hline \multirow[t]{2}{*}{ GJ $1245 \mathrm{ABC}$} & 15.18 & M5.5 V & 4.54 & $\mathrm{~S}$ & 0.198200 & 0.016990 & 331.0 & 797 & 27.47 \\
\hline & & & & $\mathrm{H}$ & 0.026270 & 0.003120 & 6.8 & 2849 & 27.19 \\
\hline \multirow[t]{2}{*}{ LHS 288} & 15.61 & M5.5 & 4.49 & $\mathrm{P}$ & 0.197300 & 0.016300 & 302 & 730 & 27.46 \\
\hline & & & & $\mathrm{H}$ & 0.00079 & 0.00028 & $11.7^{1}$ & 12927 & $25.66^{2}$ \\
\hline LHS 292 & 17.32 & M6.5 V & 4.54 & $\mathrm{P}$ & 0.006161 & 0.001330 & 39.0 & 5146 & 25.96 \\
\hline LP 71-82 & 14.43 & $\mathrm{dM} 7 \mathrm{e}$ & 5.00 & S & 0.143900 & 0.004378 & 2290.0 & 9529 & 27.41 \\
\hline LP 816-60 & 12.71 & M & 5.49 & $S$ & $<0.02430$ & & 1.9 & 326 & 26.72 \\
\hline LP 944-20 & 18.25 & M9 V & 4.97 & $\mathrm{H}$ & $<0.00415$ & & & 220800 & $25.57^{3}$ \\
\hline LTT 17897 & 12.69 & M3.5 V & 5.37 & $\mathrm{~S}$ & 0.404000 & 0.044720 & 193.0 & 475 & 27.92 \\
\hline L 34-26 & 12.90 & dM5 e & 5.99 & S & 0.594400 & 0.070090 & 171.0 & 753 & 28.18 \\
\hline DENIS 1048-39 & 18.50 & M9 V & 4.10 & S & $<0.01599$ & & 0.5 & 409 & 26.29 \\
\hline
\end{tabular}

Given value is the source likelihood.

Source not included in the HRI catalog. See Sect. 5.4.2 for details.

X-ray data taken from Neuhäuser et al. (1999). 
are derived from survey observations with correspondingly relatively low sensitivity. There is no reason to expect that these stars will not be detected once more sensitive X-ray observations are available. In other words, the formation of X-ray emitting coronae appears to be universal for late-type main sequence stars.

\subsection{Comparison to previous work}

The most complete previous compilations of X-ray data on nearby late-type stars are due to Schmitt (1994) for M and K-type stars (Table 1) and to Schmitt (1997) for F and Gtype stars (Table 2). Compared to Table 1 presented by Schmitt (1997) we first note that now the HIPPARCOS distance scale has been used. As a result of this some stars previously thought to be located within $13 \mathrm{pc}$ are no longer within that distance limit. Specifically, the stars G1 55, G1 95, Gl 97, Gl 327, Gl 364, Gl 512.1, Gl 534.1A, Gl 611A, Gl 691 and Gl 805 had to be removed from the sample. Further, the binary system G1 107AB has been observed with the HRI and the individual components are now separated; note that Gl 107B is of type M and is not included in our nearby sample because of its distance. Also, instead of upper limits we now have detections (with the ROSAT HRI) for the G-type stars G1 53A and Gl 442A. The $\mathrm{M}$ star G1 53B has also been detected but is again not member of our nearby stellar sample because of distance, while Gl 442B $(=\mathrm{VB} 5)$ at a distance of $9.24 \mathrm{pc}$ has not been detected in a $10 \mathrm{ksec}$ ROSAT HRI pointing. Schmitt et al. (1995) had complete $\mathrm{K}$ star detections only out to $7 \mathrm{pc}$, now the limit is $12 \mathrm{pc}$; on the other hand, the distance limit for the M-type stars had to be reduced to $6 \mathrm{pc}$. Including the stars between 6 and $7 \mathrm{pc}$ would add another 27 stars (from which, however, 7 remain undetected) and therefore significantly enlarge the number of undetected M-stars.

\subsection{X-ray luminosity}

In order to provide an overview over the X-ray luminosities of our sample stars we show in Fig. 6 the measured X-ray luminosity as a function of absolute magnitude $M_{V}$. Note that the range in X-ray luminosity for this sample is about three orders of magnitude essentially independent of spectral type, while the median X-ray luminosity is decreasing with increasing $M_{V}$. No objects are found in the right upper corner (there are no super-saturation $\mathrm{M}$ dwarfs) nor in the lower left corner, i.e., there are no X-ray dark solar-like stars. The dependence of median X-ray luminosity on spectral type suggests to take out the scale effect introduced by stars of different size by considering instead the mean X-ray surface flux $F_{\mathrm{X}}$ obtained by dividing $L_{X}$ by the stellar surface as in Schmitt (1997).

A mean quantity such as $F_{\mathrm{X}}$ is little meaningful for "local" structures such as individual loops or active regions, however, for "global" structures such as coronal holes, it is in fact a useful and even basic quantity. The plot of $F_{\mathrm{X}}$ vs. $M_{V}$ for our sample stars is shown in Fig. 7; as in Fig. 6, the three different types of symbols represent the F/G-type stars (plusses), K-type stars (asterisks) and M-type stars (crosses). Figure 7 indicates that the mean surface flux distributions of the three classes of stars show little difference. Possibly the mean surface flux distribution of the very faintest stars is somewhat larger on average, there is some hint that for stars with $M_{V}<13$ the lower envelope on $F_{\mathrm{X}}$ may actually increase. Also note that the K-star region now appears well filled, while there was a "gap" because of lacking sample size in the previously published data (cf., Fig. 8 in Schmitt 1997). The formal cumulative distribution functions are shown in Fig. 8, showing that indeed the means and lower envelopes of the three distribution functions agree with each other with statistical accuracy. Carrying out formal testing with Smirnov test shows that the null hypothesis that all three classes of stars are characterized by the same mean $\mathrm{X}$-ray surface flux distribution function cannot be rejected and is hence consistent with the data. We therefore conclude that not only do all cool (dwarf) stars have coronae, but that also these coronae have mean X-ray fluxes of at least $10^{4} \mathrm{erg} / \mathrm{cm}^{2} / \mathrm{s}$. We mention that these limits are grossly violated (a) by A-type stars, and (b) giants. For example, for Vega $\left(R=2.5 R_{\odot}\right)$ one would compute $L_{X}=4 \times 10^{27} \mathrm{erg}$ s; interpreting the upper limit of $1.2 \times 10^{-3} \mathrm{cts} / \mathrm{s}$, that Schmitt (1997) attributes to UV contamination, as X-ray flux, one obtains $L_{\mathrm{X}} \sim 5.5 \times 10^{25} \mathrm{erg} \mathrm{s}$, i.e., two orders of magnitude lower than "expected", and for Arcturus $\left(R=25 R_{\odot}\right)$ one obtains $L_{\mathrm{X}} \sim 4 \times 10^{29} \mathrm{erg} \mathrm{s}$, four orders of magnitude higher than the observed upper limit of $3 \times 10^{25}$ erg s (Ayres et al. 1991). The physics of coronal formation in those stars must be very different and in all likelihood those two stars are devoid of any corona.

\subsection{Time variability}

\subsubsection{Variability between survey and pointing data}

For a rather large sample of stars multiple X-ray detections are available from the RASS data and the ROSAT PSPC pointing program, which are all listed in the NEXXUS data base. In Fig. 9 we plot the PSPC count rate observed during the allsky survey vs. the PSPC count rate observed during the pointing program in a double-logarithmic representation for those of our sample stars detected in both observing modes; the two long-dashed lines delineate a factor of two deviation above and below unity. As is obvious from Fig. 9, except for four stars, all measurements lie within those dashed lines. The time span between survey and pointed observations varies considerably from star to star, but is typically of the order of 1-2 years. Obviously, variations by more than a factor of 4 are unusual at least on that time scale. The stars EV Lac, Proxima Cen and YZ Cet, all of which are well known flare stars, have significantly larger survey count rates. In the case of EV Lac this is due to a major flare which occurred during the survey observations (Schmitt 1994), the RASS light curve of Proxima Cen is discussed by Fleming et al. (1993), the RASS data on YZ Cet is discussed by Tsikoudi et al. (2000). In the case of $\zeta$ Dor (spectral type F7) the count rate observed in the pointing program was significantly higher than during the survey observations; this applies to both the PSPC observations with and without filter which had been executed immediately after each other. 


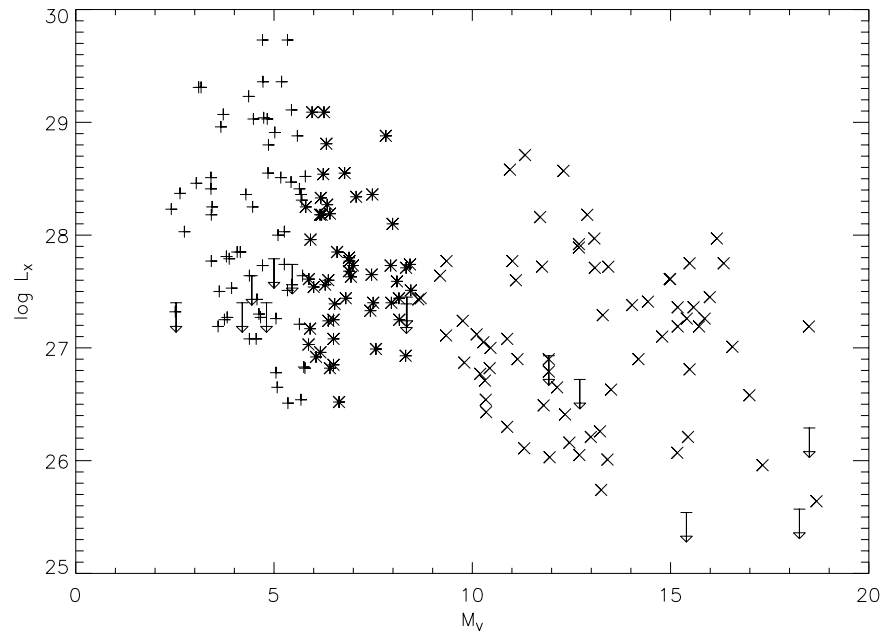

Fig. 6. X-ray luminosity vs. absolute magnitude $M_{V}$ for the nearby main-sequence stars; plusses denote F/G-type stars, asterisks K-type stars and crosses M-type stars. Note the absence of solar-like stars with X-ray luminosities of $10^{26} \mathrm{erg} / \mathrm{s}$ and below.

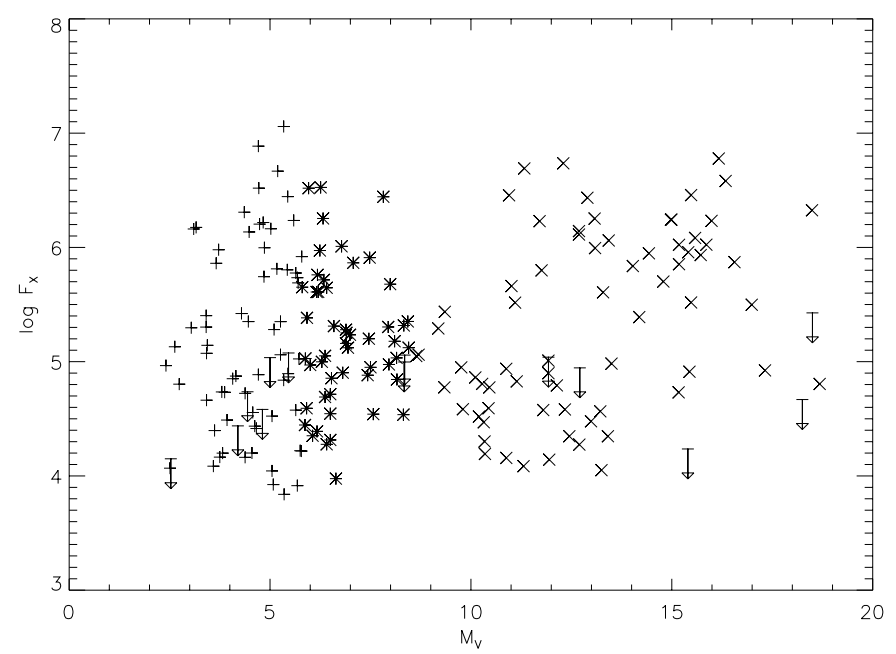

Fig. 7. Mean X-ray surface flux vs. absolute magnitude $M_{V}$ for the nearby main-sequence stars; plusses denote F/G-type stars, asterisks K-type stars and crosses M-type stars. Note the lower limit of about $10^{4} \mathrm{erg} / \mathrm{cm}^{2} / \mathrm{s}$ of observed X-ray surface flux.

In Fig. 10 we plot the PSPC pointing mode count rates measured with and without the boron filter for those NEXXUS stars observed with these instrumental setups. Observations with this setup were typically (albeit not always) taken immediately adjacent to each other, so that the time span between the data sets for a given stars should usually be of the order of a few hours. Of course, the spectral response of the PSPC detector with and without the boron filter differs especially at soft X-ray energies below the carbon edge, yet, one observes a well defined regression curve extending over two orders of magnitude. The only star far away from the regression line is again the well known flare star Proxima Cen, which clearly flared during the boron filter observation.

In Fig. 11 we plot the PSPC count rate measured in pointing program data vs. the HRI count rate in a double-logarithmic representation for those of the NEXXUS stars detected in both

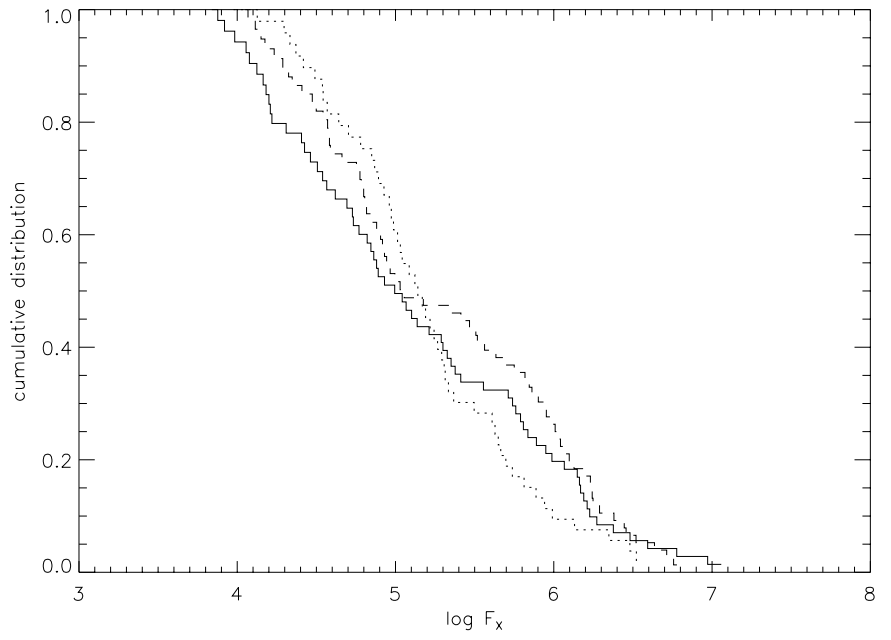

Fig. 8. Cumulative distribution function of the mean X-ray surface fluxes for F/G-type stars (stepped curve), K-type stars (dotted stepped curve) and M-type stars (dashed stepped curve). Note the close resemblance of the curves with indistinguishable mean and minimum.

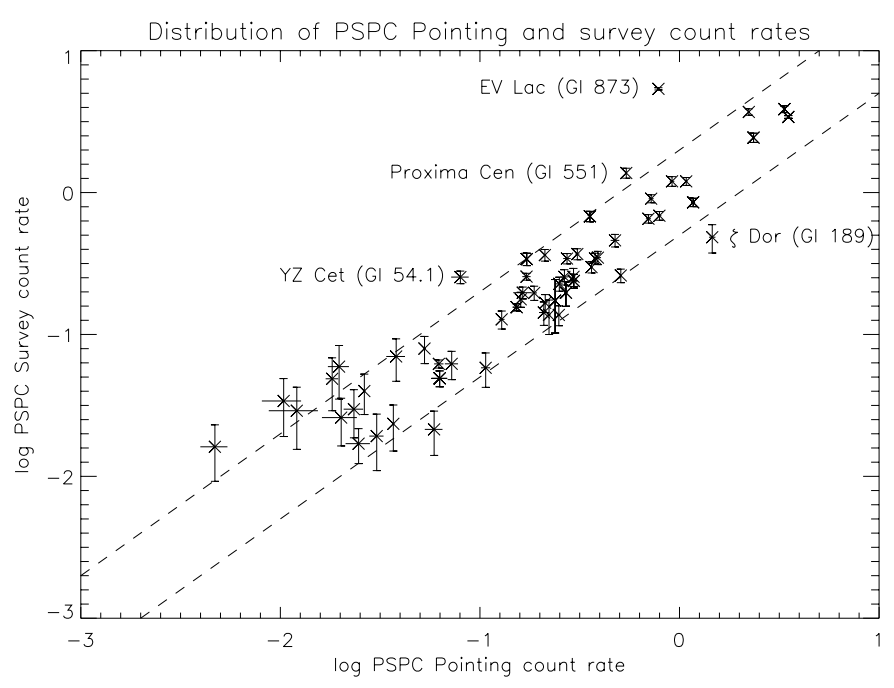

Fig. 9. Comparison of X-ray count rates for sample stars detected both in the all-sky survey and the PSPC pointing program; dashed lines indicate a factor 2 variation from unity and stars with large deviations from regression line are identified.

observing modes. In contrast to the boron filter data, the time span between PSPC and HRI pointed observations is much longer and is typically of the order of a few years for most stars. Also note that the spectral response of the HRI differs somewhat from that of the PSPC. Again, we see a good correlation between PSPC and HRI count rates over two orders of magnitude with a larger dispersion than observed for the comparison between boron and open PSPC data, but with similar dispersion as observed for the pointed and survey PSPC data for the same stars. Again, for multiply observed stars only the observation with the longest exposure time was taken into account to compile Figs. 10 and 11. 


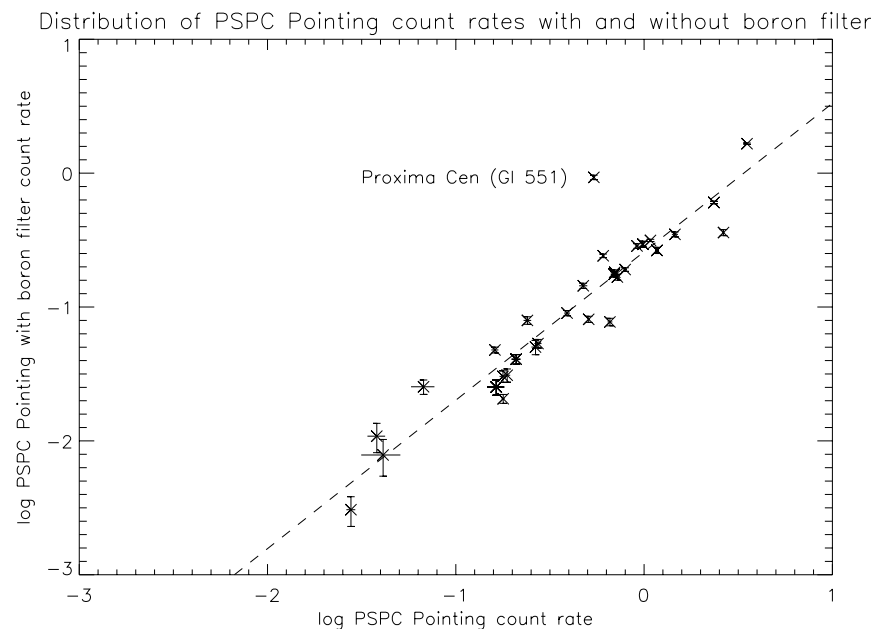

Fig. 10. Comparison of X-ray count rates for all NEXXUS stars detected in the PSPC pointing program with and without boron filter; stars with large deviations from regression line are identified.

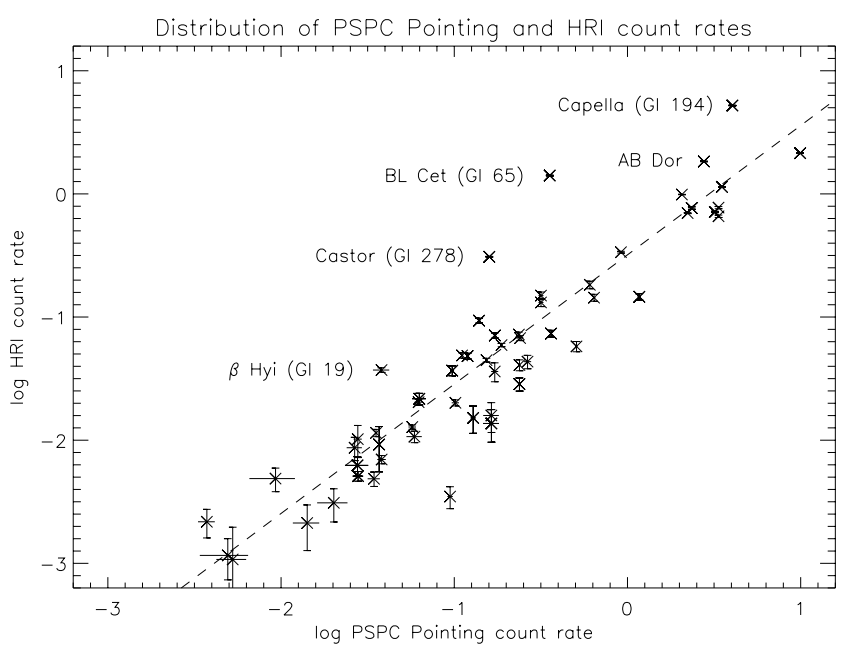

Fig. 11. Comparison of X-ray count rates for all NEXXUS stars detected both in the PSPC and HRI pointing program; stars with large deviations from regression line are identified.

\subsubsection{Variability of individual stars}

For a small number of stars repeated observations were carried out with the same instrumental setup. These datasets are discussed for the individual sources in the following section.

$\alpha$ Cen $A / B$ : The $\alpha$ Cen A/B system was observed twice with the ROSAT HRI for an extended period of time. The first set of observations was carried out in February 1996, the second one in August 1996. The obtained ROSAT HRI light curves for $\alpha$ Cen A/B are shown in Figs. 12 and 13. In both light curves the stars denote the measurements of $\alpha$ Cen B, the crosses those of $\alpha$ Cen A. As is apparent from Figs. 12 and 13, the $\mathrm{B}$ component is brighter than the A component at least every time the system was looked at with the HRI. $\alpha$ Cen A shows small daily variations, the overall level of $\mathrm{X}$ ray emission was slightly higher during the August observations. $\alpha$ Cen $\mathrm{B}$ showed considerably more X-ray variability than the A component. In February 1996 the overall emission level was higher, an excursion to more than $1 \mathrm{HRI}$ cts/s was observed on day JD 2450134 . During the second observation in August 1996 the count rate decreased by about 30 percent over

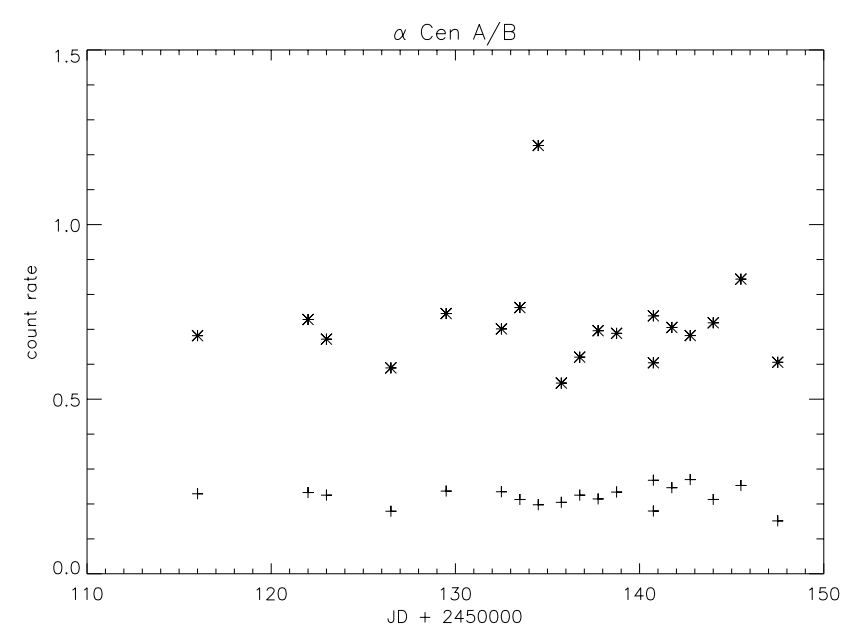

Fig. 12. X-ray light curve for $\alpha$ Cen A/B (Gl 559AB) in February 1996; asterisks denote measurements for $\alpha$ Cen B, plusses measurements for $\alpha$ Cen A.

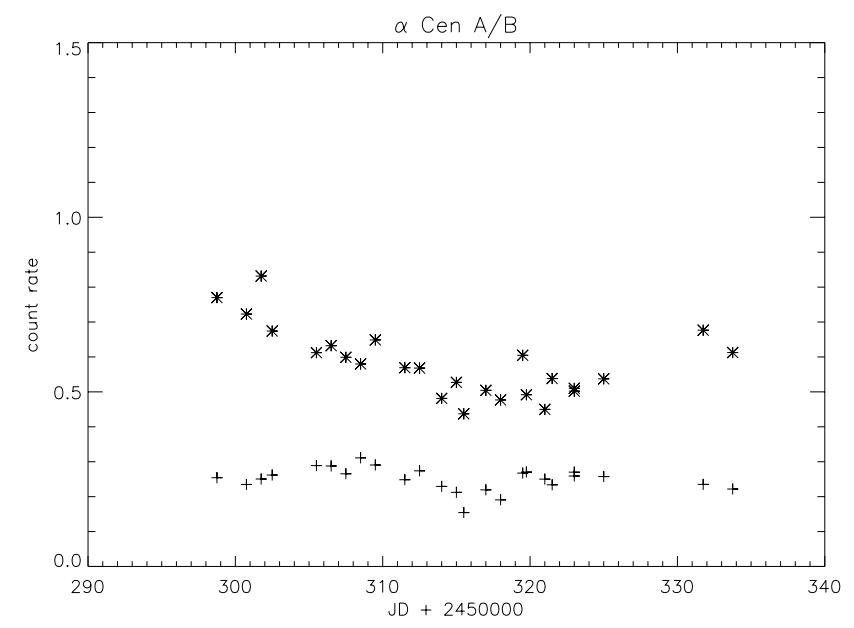

Fig. 13. X-ray light curve for $\alpha$ Cen A/B (Gl 559AB) in August 1996; asterisks denote measurements for $\alpha \mathrm{Cen} \mathrm{B}$, plusses measurements for $\alpha$ Cen A.

a 20 day interval, afterwards the count rate started increasing again. We interpret the high count rate episode in $\alpha$ Cen $\mathrm{B}$ as a flare, the 20 day decrease in count rate might be due to rotational modulation. NEXXUS lists two PSPC pointings on $\alpha$ Cen A/B, one in September 1992 for $3260 \mathrm{~s}$ and one in September 1993 for 357 s. During the September 1993 pointing the count rate was more than twice the count rate observed during the September 1992 and the RASS observations and the hardness ratio was significantly enhanced. An inspection of arrival positions of the individual photons suggests the $\mathrm{K}$ star as the source of the increased X-ray radiation in line with the light curve Fig. 12. We therefore conclude that $\alpha \mathrm{Cen} \mathrm{B}$ is a flare star.

Gl 86: The star Gl 86 was serendipitously observed during a monitoring campaign on the blazar PKS 0208-512 with the ROSAT PSPC. 12 pointings almost more or less equally spaced in time were carried out over a 13 day interval. The resulting ROSAT PSPC light curve for Gl 86 is shown in Fig. 14. The count rate first increased by almost a factor of 1.5 during the first half of the observations. After a 3-day gap the count rate started to increase in the second half again from the level it started in the first half, and then it decreased below that level. 


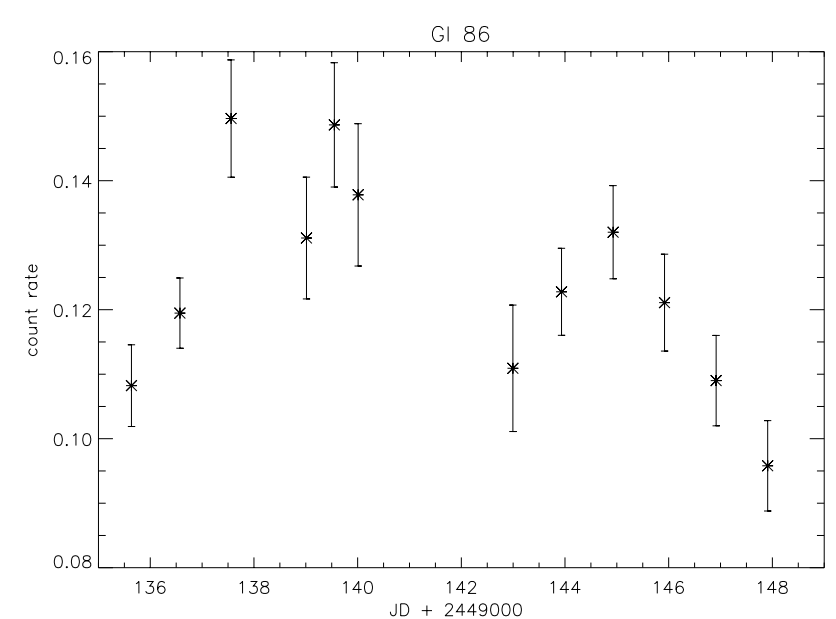

Fig. 14. X-ray light curve for Gl 86 in May/June 1993.

Variations in the X-ray output of G1 86 are quite apparent; interestingly, Marino et al. (2002) do not find variability in Gl 86 at a confidence level higher than $90 \%$ on short time scales.

Gl $820 \mathrm{~A} / \mathrm{B}$ : The visual binary $61 \mathrm{Cyg} \mathrm{A}$ and B was angularly resolved and extensively observed with the ROSAT HRI to monitor the long-term X-ray light curve of the two stars. Since these stars were also simultaneously observed in $\mathrm{Ca}$ $\mathrm{H}$ and $\mathrm{K}$, these synoptic data is presented separately by Hempelmann et al. (2003).

LHS 288: A special note is appropriate for the star LHS 288. Schmitt et al. (1995) report an upper limit of $<0.01 \mathrm{cts} / \mathrm{s}$ at the position of LHS 288 from the ROSAT allsky survey data, while Marino et al. (2000) report a detection of LHS 288 from a PSPC pointed observation. The count rate level of $0.19 \mathrm{cts} / \mathrm{s}$ reported by Marino et al. (2000) would have been easily detectable in the RASS data, however, the position of the X-ray source reported by Marino et al. (2000) does not agree with expected optical position of LHS 288 in CNS4 or in Luyten's catalog. Investigating this discrepancy we realized that Bakos et al. (2002) were unable to find and confirm even the existence of LHS 288 in their systematic attempt to follow-up high proper motion stars listed in ths LHS catalog. We therefore decided to investigate the DSS images in the $R$ and $V$ bands in the vicinity of the expected position of LHS 288. We compared the DSS images taken at epochs 1987.0512 and 1991.1096, searched for moving objects and found evidence only for one object with significant proper motion, which turned out to be also very red. The 1987.0512 position of this object is RA 10:44:22.11, $\delta-61: 12: 55.2$ (w.r.t. equinox 2000). The position of the X-ray source reported by Marino et al. (2002) is RA 10:44:21.9 and $\delta-61: 12: 44$ at epoch 1993.5575 (w.r.t. equinox 2000). According to CNS4, the proper motion of LHS 288 is 1.66 arcsec/year almost due north. Applying the proper motion of LHS 288 as listed in CNS4 to the position derived for the red moving DSS object results in almost perfect agreement with the PSPC X-ray position. We therefore conclude that the red moving DSS object is the X-ray source reported by Marino et al. (2000), and that this object is identical with LHS 288. Thus, LHS 288 has been re-found and does exist. We emphasize that also at the new position no X-ray source can be found in the RASS data with an upper limit of $<0.03 \mathrm{cts} / \mathrm{s}$.
In August 1996 a 12927 s pointed observation was carried out on LHS 288 with the ROSAT HRI. Although the automatic analysis did not show any X-ray source at the correct position of LHS 288 and consequently an appropriate entry in the ROSAT source catalogs is missing, a visual inspection of the ROSAT HRI image and a re-analysis of the individual X-ray photons revealed the presence of a weak, but still significant source at the position RA 10:44:20.99, $\delta-61: 12: 38.3$ in perfect agreement with the previously derived (and proper-motioned) PSPC and DSS positions. The observed count rate of LHS 288 as observed in August 1996 was $\approx 7.9 \times 10^{-4} \mathrm{HRI}$ cts/s; applying a factor of 4 to convert to an equivalent PSPC count rate (cf. Fig. 11), we find an equivalent PSPC rate of $\approx 3.2 \times 10^{-3} \mathrm{PSPC}$ cts/s, which is clearly consistent with the survey non-detection of LHS 288, but not with the detection of LHS 288 in the pointing program. We thus conclude that during the short PSPC pointing on July 21, 1993 the X-ray flux of LHS 288 was almost two orders magnitude larger than during the RASS observations and during the HRI pointing in August 1996, and that the PSPC observations on July 21, 1993 caught LHS 288 in an unusual state.

\section{Conclusions}

This paper attempts to provide a final and hopefully definitive summary of the ROSAT observations of nearby stars. It extends our previous studies of the subject and the now presented source tables supersede those published previously. One of our main results is the universality of $\mathrm{X}$-ray emission among latetype stars with outer convection zones. As to the F/G-type stars, all stars at distances below 12 pc have been detected, all the upper limits obtained in the distance range $12-14$ pc result from the lower sensitivity survey data. As to the K-type stars, all stars at distances below $8 \mathrm{pc}$ have been detected and only two stars in the distance range 8-12 pc remain undetected; two of those upper limits come from survey data, the other one is derived from a rather short ROSAT HRI pointing. As to the M-type stars, only two stars in the volume out to $6 \mathrm{pc}$ remain undetected if very late-type stars and brown dwarfs are excluded from consideration; one upper limit comes from survey data, one from a short PSPC pointing. If all known stars within 6 pc are included, the number of non-detected stars increases to 6 ; in one case (LP944-20) a Chandra detection of such an object during a flare has been obtained. We therefore conclude that all mainsequence stars with outer convection zones are surrounded by hot coronae and that the reason for our not being able to detect all stars is lack of sufficient sensitivity rather than the intrinsic absence of X-ray emission. X-ray dark cool stars on the main sequence do not exist.

The X-ray luminosities of cool dwarf stars extend over three orders of magnitude with the mean values decreasing with decreasing spectral type. Scaling the X-ray luminosity with the stellar surface results in an activity measure $F_{X}$ independent of spectral type with essentially indistinguishable distribution functions. In particular, stars with the lowest degree of activity in any subclass always have mean X-ray surface fluxes of $\approx 10^{4} \mathrm{erg} / \mathrm{cm}^{2} / \mathrm{s}$. Schmitt (1997) demonstrated that this flux level is approximately the one attained by solar coronal holes, the much larger sample available now confirms this conclusion. 
Of course, there is no direct proof that the X-ray emission originates from magnetically open (rather than closed) regions in those stars. However, the fact that the lower surface flux level is so similar from F-type stars through to M-type stars as well as the lack of observed variability suggest a global rather than a local property as cause of the observed similarities. As an interesting aside we note that for a star like the Sun the X-ray luminosity contained in this "coronal hole"-component amounts to approximately $6 \times 10^{26} \mathrm{erg} / \mathrm{s}$ formally in the $0.1-2 \mathrm{keV}$, with actually almost all flux contained in the $0.1-0.5 \mathrm{keV}$ band because of the low X-ray temperatures (cf., Fig. 7 in Schmitt 1997). Relating this value to the bolometric luminosity via $L_{\mathrm{X}, \mathrm{CH}} / L_{\mathrm{bol}}$ results in a value of approximately $1.5 \times 10^{-7}$, i.e., the same value found to describe the X-ray emission from early type stars (which is also thought to arise in winds). It is unclear whether this agreement is simply a numerical coincidence or indicative of a physical connection.

The question of X-ray emission among the very latest mainsequence stars and brown dwarfs remains unclear. ROSAT observations usually do not have the sensitivity level to reach the required surface flux levels of $10^{4} \mathrm{erg} / \mathrm{cm}^{2} / \mathrm{s}$ at the stellar surface; for a typical star at the bottom of the main sequence this corresponds to an X-ray luminosity of $6 \times 10^{24} \mathrm{erg} / \mathrm{s}$ (in the $0.1-0.5 \mathrm{keV}$ band) which is hard to detect even for stars in the immediate solar vicinity. More importantly, the observed large amplitudes of X-ray variability as seen for vB10 (=G1752B; Fleming et al. 2000), LHS 2065 (Schmitt \& Liefke 2002), LP944-20 (Rutledge et al. 2000) and for LHS 288 (this paper) suggest that the physics of coronal formation for very low mass stars and brown dwarfs may be different and X-ray emission may be present only in a transient fashion.

This behavior with large amplitude variability has to be juxtaposed to the the relatively small degree of X-ray variability found for stars of low activity levels in extensive ROSAT observations. Comparison of stars with multiple PSPC observations (in particular for stars with both survey and pointing detections available) shows that for most stars the two data sets are within a factor of two from unity; the only stars with discrepant fluxes are known flare stars, where the observed count rate excursions can be clearly attributed to flare events. The Sun is known to vary its X-ray output during a solar cycle by almost two orders of magnitude (Acton 1996), however, the observed amplitude of variability strongly depends on the spectral band considered. The $\mathrm{YOHKOH}$ data described by Acton (1996) refer to a somewhat harder X-ray band than the typical ROSAT data are referred to, and therefore a solar peak-to-peak variation in the ROSAT band of a factor 100 during a solar cycle seems too extravagant an expectation. At any rate, from the ROSAT observations we can state that count rate variations usually stay within a factor of 4 with exceptions attributable to flares. So, either the stellar variability level is lower or the time full time scale of variability has not been adequately sampled by ROSAT.

Acknowledgements. The ROSAT work on nearby stars would have been impossible without the ROSAT all-sky survey. We thank H. Jahreiss for making available to us his latest version of CNS4. We have made extensive use of the ROSAT Data Archive of the Max-Planck-Institut für extraterrestrische Physik (MPE) at Garching,
Germany. Also, this research has made use of the SIMBAD database, operated at CDS, Strasbourg, France. We particularly thank Dr. J. Pye for providing us with the ROSAT-WFC data for inclusion into the NEXXUS data base. We thank our many colleagues for numerous discussions on the X-ray properties of nearby stars.

\section{References}

Acton, L. 1996, in Cool Stars, Stellar Systems, and the Sun, ASP Conf. Ser., 109, 45

Ayres, T. R., Fleming, T. A., \& Schmitt, J. H. M. M. 1991, ApJ, 376, L45

Bakos, G. Á., Sahu, K. C., \& Németh, P. 2002, ApJS, 141, 187

Berghöfer, T. W., Schmitt, J. H. M. M., Danner, R., \& Cassinelli, J. P. 1997, A\&A, 322, 167

Delfosse, X., Forveille, T., Martín, E. L., et al. 2001, A\&A, 366, L13

Fleming, T. A., Giampapa, M. S., \& Schmitt, J. H. M. M. 2000, ApJ, 533,372

Fleming, T. A., Giampapa, M. S., Schmitt, J. H. M. M., \& Bookbinder, J. A. 1993, ApJ, 410, 387

Fleming, T. A., Snowden, S. L., Pfeffermann, E., Briel, U., \& Greiner, J. 1996, A\&A, 316, 147

Fuhrmeister, B., \& Schmitt, J. H. M. M. 2003, A\&A, 403, 247

Hempelmann, A., Schmitt, J. H. M. M., Baliunas, S. L. \& Donahue, R. A. 2003, A\&A, 406, 39

Hünsch, M. 2001, in 11th Cambridge Workshop on Cool Stars, Stellar Systems and the Sun, ASP Conf. Ser., 223, 967

Hünsch, M., Schmitt, J. H. M. M., Sterzik, M. F., \& Voges, W. 1999, A\&AS, 135, 319

Haisch, B., \& Schmitt, J. H. M. M. 1994, ApJ, 426, 716

Hünsch, M., Schmitt, J. H. M. M., \& Voges, W. 1998a, A\&AS, 127, 251

Hünsch, M., Schmitt, J. H. M. M., \& Voges, W. 1998b, A\&AS, 132, 155

Jahreiss, H. 2002, private communication

Marino, A., Micela, G., \& Peres, G. 2000, A\&A, 353, 177

Marino, A., Micela, G., Peres, G., \& Sciortino, S. 2002, A\&A, 383, 210

Neuhäuser, R., Briceño, C., Comerón, F., et al. 1999, A\&A, 343, 883

Pye, J. P., McGale, P. A., Allan, D. J., et al. 1995, MNRAS, 274, 1165

Randich, S. 2000, in Stellar Clusters and Associations: Convection, Rotation, and Dynamos, ASP Conf. Ser., 198, 401

Rutledge, R. E., Basri, G., Martín, E. L., \& Bildsten, L. 2000, ApJ, 538, L141

Schmitt, J. H. M. M. 1994, ApJS, 90, 735

Schmitt, J. H. M. M. 1997, A\&A, 318, 215

Schmitt, J. H. M. M., Fleming, T. A., \& Giampapa, M. S. 1995, ApJ, 450,392

Schmitt, J. H. M. M., Golub, L., Harnden, F. R., et al. 1985, ApJ, 290, 307

Schmitt, J. H. M. M., Kahabka, P., Stauffer, J., \& Piters, A. J. M. 1993, A\&A, 277, 114

Schmitt, J. H. M. M., \& Liefke, C. 2002, A\&A, 382, L9

Scholz, R.-D., Meusinger, H., \& Jahreiß, H. 2001, A\&A, 374, L12

Schröder, K.-P., Hünsch, M., \& Schmitt, J. H. M. M. 1998, A\&A, 335, 591

Simon, T., Drake, S. A., \& Kim, P. D. 1995, PASP, 107, 1034

Stern, R. A., Schmitt, J. H. M. M., \& Kahabka, P. T. 1995, ApJ, 448, 683

Sterzik, M. F., \& Schmitt, J. H. M. M. 1997, AJ, 114, 1673

Tsikoudi, V., Kellett, B. J., \& Schmitt, J. H. M. M. 2000, MNRAS, 319,1136

Vaiana, G. S., Fabbiano, G., Giacconi, R., et al. 1981, ApJ, 245, 163 